\title{
A Quasi-Continuum Thermomechanical Model for Phonon Damping Analysis of Single Crystal Silicon Nano-Resonators
}

\author{
Y. Yu, H. Zhao and G. Li* \\ Department of Mechanical Engineering \\ Clemson University, Clemson, SC 29634-0921
}

\begin{abstract}
In this paper, we study phonon-mediated intrinsic damping in single crystal silicon nano-resonators where the phonon transport is of partial ballistic and partial diffusive nature. For this purpose, we present a quasicontinuum thermomechanical model that accounts for both thermoelastic and Akhiezer energy dissipation mechanisms. In the model, the linearized frequency-dependent phonon Boltzmann transport equation (BTE) is coupled with continuum elasticity equations via phonon modulation theory. The model is implemented numerically by using the finite element and finite volume methods for calculating the damping ratio and quality factor of nano-resonators under forced vibration. Both flexural and axial motions are considered for single crystal silicon nano-resonators with different sizes and frequencies. The numerical results obtained from the quasi-continuum thermomechanical model are compared with those obtained from molecular dynamics simulations.
\end{abstract}

Keywords: Damping, phonon transport, thermomechanical model, nano-resonators, quality factor

*Author to whom correspondence should be addressed. Electronic mail: gli@clemson.edu 


\section{Introduction}

The quality factor of nano-resonators, which is characterized by the ratio of energy stored to energy loss over a period of operation time, plays an important role in the performance and stability of nano-electromechanical devices [1]. In general, energy dissipation in nano-resonators can be categorized into two types: extrinsic dissipation and intrinsic dissipation. Extrinsic dissipation includes damping caused by the surrounding environment [2], material impurities [3], and structural support [4]. This type of energy dissipation can be reduced or even eliminated through optimized engineering design and manufacturing process. On the other hand, intrinsic dissipation is due to the system's inherent properties. For mechanical resonators, intrinsic dissipation mainly includes thermoelastic damping (TED) [5], Akhiezer damping [6] and surface damping [7]. TED occurs when a structural motion induced inhomogeneous strain field results in internal spatial heat flow, and hence thermal energy dissipation. TED is relatively well understood for micro-resonators. The classical TED theory $[5,8,9]$ predicts a single-peak Lorentian behavior of the thermoelastic damping ratio in the frequency domain. Khisaeva [10] proposed a TED theory with second sound effects by adding the derivatives of heat flux into Fourier's law, which captures non-diffusive (hyperbolic decaying) heat flow effects. Solutions of the modified theory show that the thermoelastic damping ratio contains many other peaks over the frequency domain $[10,11]$. While these models have been successfully applied to micro-resonators, they are not directly applicable to nano-resonators due to the fact that the heat flow in nanoscale structures is partially diffusive and partially ballistic in nature. In addition to TED, Akhiezer damping takes place due to the frequency-dependent phonon modulation caused by the same strain field and, consequently, the internal heat flow between the perturbed phonon groups of different frequencies. Such non-spatial but phonon-frequencyrelated heat redistribution leads to further energy dissipation. Akhiezer damping has received much attention in studies of nano-resonators, and has been compared with TED in terms of their relative importance. A number of research works including theoretical analysis [6,12], experiments [13,14], and molecular dynamics (MD) simulations [14] have concluded that the role of Akhiezer damping becomes significant when the resonator size becomes smaller than the phonon mean free path.

The thermal transport and dissipation mechanisms become different when the size of spatial domain reduces to nanoscale and the frequency of vibration reaches gigahertz level. For thermal transport, both Fourier's law and its modification with second sound effect have been shown to be inadequate in the nano-regime [15]. Instead, the phonon Boltzmann transport equation (BTE) model has been applied to thermal transport analysis in various nano-scale applications with demonstrated accuracy and efficiency $[16,17,18]$, mainly because it 
views heat conduction as collective interactions of phonons and captures both diffusive and ballistic behavior of phonon transport through various scattering mechanisms. The BTE treatment in nano-scale thermal transport suggests that the intrinsic damping can be theorized in the light of phonon-mediated energy dissipation, in which the coupling between strain and phonons redefines the phonon distribution, transport and relaxation, and hence energy dissipation. With the BTE treatment in damping analysis, it is important to acknowledge that phonon distribution and transportation are spatial and frequency dependent. By incorporating the spatial dependence in the BTE model, one evaluates the coupling between the spatially inhomogeneous elastic and phonon fields, hence the nano-scale equivalence of TED. On the other hand, by incorporating the frequency dispersion in the BTE model, one evaluates the strain-induced local relaxation between phonon branches/modes, hence the Akhiezer damping. Altogether, the phonons are modulated, both spatially and between dispersion branches, by the strain field through the generalized frequency dependent Grüneisen tensor. The fundamental assumption is that the coupling between elastic strain field and phonon distribution can be established through the phonon modulation theory [6, 19]. Several authors [20, 21, 22] have calculated the mode-dependent Grüneisen parameters that represent the strength of influence. In the light of this, Kiselev and Iafrate [23] developed a semi-analytical phonon-modulation damping model for flexural nano-beams and provided a qualitative view of the damping behavior as a function of beam length, aspect ratio and vibration frequency. However, the semi-analytical model is based on significant theoretical simplifications such as 1-D Euler-Bernoulli beam theory, the Debye model for phonon spectrum and 1-D thermal transport assumption. In addition, the strength of strain-phonon interaction is represented by only two distinctive Grüneisen parameters for the entire phonon dispersion. Kunal and Aluru [14] have recently investigated the Akhiezer damping of nano-bars using MD simulations and provided a clearer view of its relative importance as compared to surface damping. However, the atomistic simulation methods such as MD are cumbersome for damping analysis of nano-resonators due to the high computational cost in tracing time history of the dynamic response.

The above issues lead to the motivation of formulating the holistic damping mechanism, via the phonon modulation theory, into a coupled thermomechanical computational model for numerical analysis of intrinsic damping in crystalline nano-structures. In this paper, we present a quasi-continuum thermomechanical model for this purpose. It should be noted that, while the proposed model is applied to the intrinsic damping analysis of single crystal silicon nano-resonators in this paper, it is a general model and applicable to other crystalline materials. In the general framework of the model, lattice dynamics is adopted to calculate thermodynamic properties of the crystalline material such as phonon dispersion, mode-dependent Grüneisen parameters and elastic constants. With these properties as parameters, the phonon transport is described by 
frequency-dependent BTE to account for details of the phonon dispersion and phonon-phonon scatterings. This provides the treatment of the phonon redistribution in the Akhiezer damping. The strain field in the nano-structure is calculated by using the continuum elasticity theory considering thermal transport induced stress corrections. Furthermore, elastic scattering and structural boundary scattering of phonons are included in the BTE model. In short, the phonon BTE model is coupled with the continuum elasticity model through strain-induced phonon modulation and phonon transport induced stress corrections. The coupled thermomechanical model is solved numerically to obtain the quality factor $Q$ of nano-resonators. The finite element method (FEM) is employed for the discretization of the mechanical equation of motion. The finite volume method (FVM) is used for the discretization of the phonon BTE. Both flexural and axial motions are considered for nano-resonators with different lengths and frequencies. The numerical results obtained from the quasi-continuum thermomechanical model are compared with those obtained from MD simulations.

The rest of the paper is organized as follows. Section 2 illustrates the theoretical framework of the phononmediated damping theory. In Section 3, the numerical schemes and solution methods are described in details. Numerical results are presented in Section 4. Finally, Section 5 gives the conclusions.

\section{Theory}

\subsection{Thermodynamic Properties of Crystals}

At the atomistic level, the interaction between atoms in the crystal lattices can be described by empirical interatomic potentials such as the Tersoff [24], Brenner [25] and Stillinger-Weber [26] potentials. Tersoff empirical interatomic potential is employed in this work for Si. Typically, the total potential energy $U$ of a $\mathrm{N}$-atom system is given by,

$$
U=\sum_{\alpha} U_{\alpha}=\frac{1}{2} \sum_{\alpha \neq \beta} V_{\alpha \beta}
$$

where $\alpha$ and $\beta$ are the atoms of the system and $V_{\alpha \beta}$ is the bond energy between atoms $\alpha$ and $\beta$. For a crystal lattice under deformation, the phonon dispersion can be obtained by computing the eigenvalues of the deformation dependent dynamical matrix $\mathbf{D}(\mathbf{k})$ for each wave vector $\mathbf{k}$ in the first Brillouin zone [27]

$$
\mathbf{D}(\mathbf{k})=\frac{1}{M}\left[\begin{array}{ll}
\sum_{\beta} \boldsymbol{\Phi}_{j, k}^{11}(\alpha, \beta) e^{i \mathbf{k} \cdot \mathbf{R}_{\beta \alpha}^{0}} & \sum_{\beta} \boldsymbol{\Phi}_{j, k}^{12}(\alpha, \beta) e^{i \mathbf{k} \cdot\left(\mathbf{R}_{\beta \alpha}^{0}-\mathbf{F}^{-1} \boldsymbol{\xi}\right)} \\
\sum_{\beta} \boldsymbol{\Phi}_{j, k}^{21}(\alpha, \beta) e^{i \mathbf{k} \cdot\left(\mathbf{R}_{\beta \alpha}^{0}+\mathbf{F}^{-1} \boldsymbol{\xi}\right)} & \sum_{\beta} \boldsymbol{\Phi}_{j, k}^{22}(\alpha, \beta) e^{i \mathbf{k} \cdot \mathbf{R}_{\beta \alpha}^{0}}
\end{array}\right](j, k=1,2,3)
$$


where $M$ is the mass of atom, $\mathbf{k}$ is the wave vector, $\mathbf{R}_{\beta \alpha}^{0}$ is the vector between the equilibrium positions of atoms $\alpha$ and $\beta, \mathbf{F}$ is the deformation gradient, $\boldsymbol{\xi}$ is the inner displacement between the 2 Bravais lattices, and $\Phi$ denotes the force constants which are the second derivatives of the potential energy with respect to the displacements of atoms $\alpha$ and $\beta$. Based on the strain dependent reciprocal space quasiharmonic model (QHMK) proposed in our previous work [27], the phonon frequencies $\omega$ can be calculated from the eigenvalues of $\mathbf{D}(\mathbf{k})$. By using the QHMK, a set of physical properties, including the dimensionless phonon frequencies $x$, phonon density of states $D(\omega)$, Helmholtz free energy $A$, mode specific heat capacity $C_{v}$, phonon group velocity $v_{g}$, mode Grüneisen parameter $\gamma_{i j}$, the second Piola-Kirchhoff stress tensor $S_{i j}$ and isothermal elastic constants $C_{i j k l}$, can also be calculated. The properties calculated from QHMK model are summarized in Table.1. The detailed calculations can be found in Refs. [27, 28].

Table 1: Phonon, thermodynamic and elastic properties calculated from QHMK

\begin{tabular}{ll}
\hline \hline Physical property & Formula \\
Dimensionless phonon frequencies $x$ & $x=\frac{\hbar \omega(\lambda, \mathbf{k})}{k_{B} T}, \quad \lambda$ : phonon branch, k: wave vector \\
Mode specific heat capacity $C_{v}$ & $C_{v}=k_{B} \frac{x^{2} e^{x}}{\left(e^{x}-1\right)^{2}}$ \\
phonon group velocity $v_{g}$ & $v_{g}=\left|\frac{\partial \omega}{\partial \mathbf{k}}\right|$ \\
Mode Grüneisen parameter $\gamma_{i j}$ & $\gamma_{i j}=-\frac{1}{\omega} \frac{\partial \omega}{\partial E_{i j}}, \quad E:$ Green-Lagrange strain \\
Helmholtz free energy $A$ & $A=U_{0}+k_{B} T \int\left(\frac{1}{2} x+\ln \left(1-e^{-x}\right)\right) D(\omega) d \omega$ \\
Second Piola-Kirchhoff stress $S_{i j}$ & $S_{i j}=\frac{\partial U_{0}}{\partial E_{i j}}-\int\left(\frac{1}{2} \hbar \omega+\frac{\hbar \omega}{e^{x}-1}\right) \gamma_{i j} D(\omega) d \omega$ \\
Elastic constants $C_{i j k l}$ & $C_{i j k l}=\frac{\partial S_{i j}}{\partial E_{k l}}$ \\
\hline
\end{tabular}

\subsection{Strain modulated Boltzmann transport equation}

In the damping analysis of nano-resonators, the phonon BTE is the governing equation of thermal transport. The phonon BTE is given in terms of phonon distribution function $f(\omega, \mathbf{r}, t)$ as [23]

$$
\dot{f}+v_{g}\left(\mathbf{s} \cdot \nabla_{\mathbf{r}} f\right)+\frac{\partial \omega}{\partial t} \frac{\partial f}{\partial \omega}=\left(\frac{\partial f}{\partial t}\right)_{\text {coll }}
$$


where $\mathbf{r}$ is spatial position, $\mathbf{s}$ is the unit directional vector of phonon velocity and the right hand side of Eq. (3) represents the phonon relaxation due to scattering. The equilibrium phonon distribution at $\omega$ and $T$ is defined by the Bose-Einstein distribution function, $f_{0}(\omega, T)=1 /\left(e^{(\hbar \omega) /\left(k_{B} T\right)}-1\right)$. In the presence of a strain field, local strain modulates phonon frequencies $\omega$ through the mode Grüneisen parameter $\gamma_{i j}$, and, consequently, distorts the phonon distribution $f$. The strain modulated phonon frequency is given by [6],

$$
\omega^{\prime}=\omega\left(1-\sum_{i j} \gamma_{i j} E_{i j}\right), \quad(i, j=1,2,3),
$$

where $\omega^{\prime}$ is the modulated phonon frequency. It should be noted that, in the nano regime, the local process is considered isothermal [23]. The strain-modulated phonons with frequency $\omega^{\prime}$ cause the phonon distribution $f$ to deviate from its original equilibrium $f_{0}$ and hence result in an irreversible heat flow towards a new equilibrium distribution $f_{0}\left(\omega^{\prime}, T^{\prime}\right)$ with a different temperature $T^{\prime}$. In this work, a small perturbation of strain fields is assumed in the damping analysis. Under this assumption, the Green-Lagrange strain $E_{i j}$ reduces to engineering strain $\epsilon_{i j}$, and a linearized approximation is employed for $f$ such that

$$
\frac{\partial \omega^{\prime}}{\partial t} \frac{\partial f}{\partial \omega^{\prime}} \approx \frac{\partial \omega^{\prime}}{\partial t} \frac{\partial f_{0}\left(\omega^{\prime}, T^{\prime}\right)}{\partial \omega^{\prime}} \approx \frac{\partial \omega^{\prime}}{\partial t} \frac{\partial f_{0}(\omega, T)}{\partial \omega} .
$$

Substituting Eq.(5) into Eq.(3), we obtain

$$
\dot{f}+v_{g}\left(\mathbf{s} \cdot \nabla_{\mathbf{r}} f\right)+\sum_{i j} \gamma_{i j} \dot{\epsilon}_{i j} \frac{x e^{x}}{\left(e^{x}-1\right)^{2}}=\left(\frac{\partial f}{\partial t}\right)_{\text {coll }},
$$

where $x=(\hbar \omega) /\left(k_{B} T\right)$. Furthermore, the phonon dispersion curves of the material is discretized into a set of energy bands. Figure 1 shows the discretization scheme of the Si dispersion curves in [100] direction. The longitudinal acoustic (LA) and transverse acoustic (TA) branches are uniformly discretized along the $\mathbf{k}$ axis. The frequency and wave vector intervals of band $n$ are denoted as $\Delta \omega_{n}$ and $\Delta k_{n}$, respectively. The optical branches are treated as one single band with a center frequency $\omega_{o}=9.0 \times 10^{13} \mathrm{rad} / \mathrm{s}$ and group velocity $\left(v_{g}\right)_{o}=0$. Thus, Eq. (6) can be re-written in terms of phonon energy densities of the discretized phonon energy bands as

$$
\dot{e}_{n}+v_{n}\left(\nabla_{\mathbf{r}} \cdot e_{n} \mathbf{s}\right)+\sum_{i j}\left(\chi_{i j}\right)_{n} \dot{\epsilon}_{i j}=\left(\frac{\partial e_{n}}{\partial t}\right)_{c o l l},
$$

The physical parameters associated with Eq. (7) are defined in Table 2. With the phonon energy densities, the effective lattice temperature $T$ at a spatial location can be defined as

$$
T=\frac{1}{C_{b u l k}} \sum_{n=1}^{N_{b}} \int_{4 \pi} e_{n} d \Omega
$$




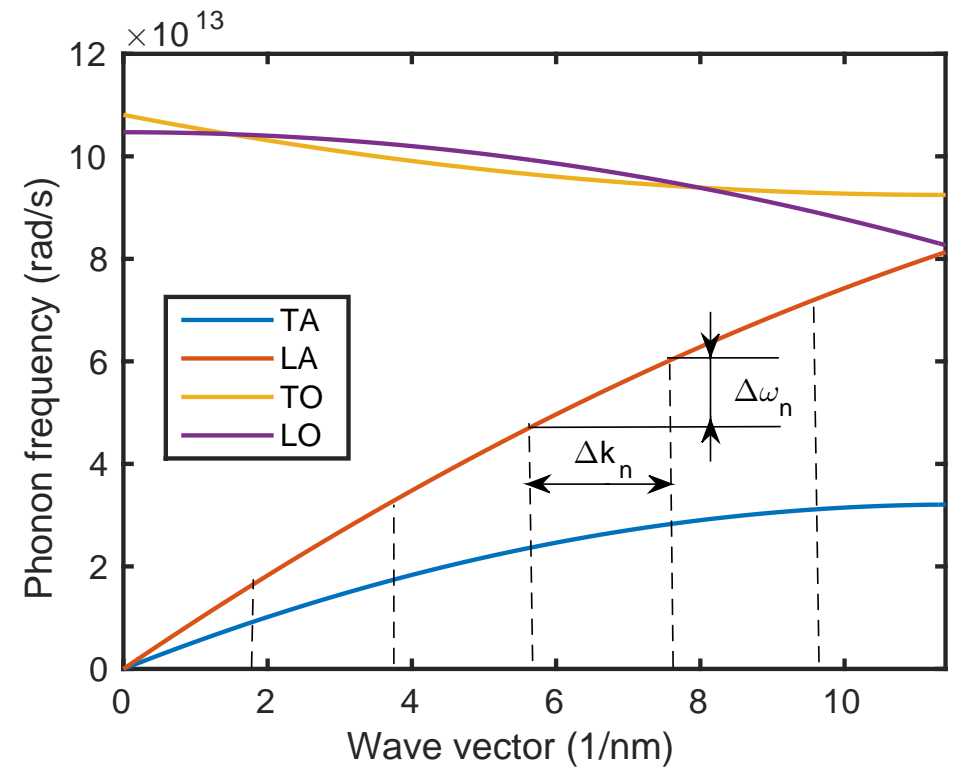

Figure 1: Discretization of [100]-direction phonon dispersion curves in into energy bands.

Table 2: Variables and phonon properties of the $n$-th energy band

\begin{tabular}{ll}
\hline \hline Property & Formula \\
Phonon energy density $e_{n}$ & $e_{n}(\mathbf{r}, \mathbf{s}, t)=\int_{\Delta \omega_{n}} \hbar \omega D(\omega) f d \omega$ \\
Equilibrium phonon energy density $e_{n 0}$ & $e_{n 0}(\mathbf{r}, t)=\frac{1}{4 \pi} \int_{4 \pi} e_{n} d \Omega$ \\
Heat capacity $\left(C_{v}\right)_{n}$ & $\left(C_{v}\right)_{n}=\int_{\Delta \omega_{n}} D(\omega) C_{v} d \omega$ \\
Group velocity $v_{n}$ & $v_{n}=\frac{1}{C_{n}} \int_{\Delta \omega_{n}} D(\omega) C_{v} v_{g} d \omega$ \\
Grüneisen parameter $\left(\gamma_{i j}\right)_{n}$ & $\left(\gamma_{i j}\right)_{n}=\frac{1}{C_{n}} \int_{\Delta \omega_{n}} D(\omega) C_{v} \gamma_{i j} d \omega$ \\
Phonon-modulating parameter $\left(\chi_{i j}\right)_{n}$ & $\left(\chi_{i j}\right)_{n}=\frac{T_{r e f}}{4 \pi} \int_{\Delta \omega_{n}} D(\omega) C_{v} \gamma_{i j} d \omega, \quad T_{r e f}$ : reference temperature \\
\hline
\end{tabular}

where $N_{b}$ is the number of energy bands, $C_{b u l k}$ is the bulk specific heat, and $\Omega$ is the solid angle.

For the phonon scattering term on the right hand side of Eq. (7), we adopt the linearized relaxation time approach from the work of Narumanchi et al [16], where $\left(\frac{\partial e_{n}}{\partial t}\right)_{\text {coll }}$ is defined as

$$
\left(\frac{\partial e_{n}}{\partial t}\right)_{\text {coll }}=\left(e_{n 0}-e_{n}\right)\left(\tau_{n n}^{-1}\right)_{a v g}+\sum_{m=1, m \neq n}^{N_{b}}\left[\frac{1}{4 \pi}\left(C_{v}\right)_{n}\left(T_{m n}-T_{r e f}\right)-e_{n}\right]\left(\tau_{m n}^{-1}\right)_{a v g}
$$


where $\tau_{m n}$ denotes the relaxation time for phonon scattering between band $m$ and band $n,\left(\tau_{m n}^{-1}\right)_{a v g}$ denotes the inverse band-averaged relaxation time, and $T_{m n}$ is defined as the interaction temperature between band $m$ and $n$ through phonon scattering. For conservation of energy, $T_{m n}$ is defined as [16],

$$
T_{m n}=T_{n m}=T_{r e f}+4 \pi \frac{e_{n 0} \Delta \omega_{m}+e_{m 0} \Delta \omega_{n}}{\left(C_{v}\right)_{n} \Delta \omega_{m}+\left(C_{v}\right)_{m} \Delta \omega_{n}} .
$$

The calculation of the band-averaged inverse relaxation time accounts for different intrinsic phonon scattering mechanisms such as phonon-impurity scattering $\left(\tau_{n n}^{-1}\right)_{I}$, Umklapp and normal phonon-phonon scattering processes $\left(\tau_{m n}^{-1}\right)_{U, N}$. The calculation is based on the following assumptions:

1. The inverse relaxation times are dependent on a number of intrinsic factors such as phonon frequency $\omega$ and group velocity $v_{g}$, as well as extrinsic factors such as temperature $T$.

2. The scattering mechanisms are independent of each other and follow Matthiessen's rule.

3. $\left(\tau_{n n}^{-1}\right)_{a v g}$ for $\mathrm{Si}$ isotope impurity scattering can be approximated based on the scattering rates on point defects as [29]

$$
\left(\tau_{n n}^{-1}\right)_{a v g}=\left(\tau_{n n}^{-1}\right)_{I}=\frac{1}{\Delta \omega_{n}} \int_{\Delta \omega_{n}} \frac{\pi}{6} \Gamma V_{0} D(\omega) \omega^{2} d \omega,
$$

where $\Gamma$ is the isotope impurity mass difference parameter and $V_{0}$ is the volume per atom. Note that, although $\left(\tau_{n n}^{-1}\right)_{\text {avg }}$ may also include other scattering mechanisms such as substitutional dopant impurity scattering [30], phonon-electron scattering [31], and others under the Matthiessen's rule, for single crystal $\mathrm{Si}$, these terms are omitted here.

4. Since, for the temperature of interest $(200 K \leq T \leq 600 K)$, the phonon relaxation and thermal resistance $[32,33]$ are mainly due to the phonon-phonon U-process, $\left(\tau_{m n}^{-1}\right)_{a v g}$ is calculated by summing up all the admissible scatterings taking place within the individual bands $\Delta \omega_{n}$ as $[34,35]$.

$$
\begin{aligned}
& \left(\tau_{n m}^{-1}\right)_{L T L}=\frac{1}{\Delta \omega_{n}} \int_{\Delta \omega_{m n}} \frac{2 \gamma_{b u l k}^{2} \hbar}{3 \pi v_{p h}^{2}\left(\omega_{n}\right) v_{g}\left(\omega_{m}\right)} \omega_{n} \omega_{T} \omega_{m} r_{c}^{2}\left(f_{0}\left(\omega_{T}\right)-f_{0}\left(\omega_{m}\right)\right) d \omega_{n} \\
& \left(\tau_{n m}^{-1}\right)_{T T L \text { or } T L L}=\frac{1}{\Delta \omega_{n}} \int_{\Delta \omega_{m n}} \frac{\gamma_{b u l k}^{2} \hbar}{3 \pi v_{p h}^{2}\left(\omega_{n}\right) v_{g}\left(\omega_{m}\right)} \omega_{n} \omega_{m} \omega_{L} r_{c}^{2}\left(f_{0}\left(\omega_{m}\right)-f_{0}\left(\omega_{L}\right)\right) d \omega_{n} \\
& \left(\tau_{n m}^{-1}\right)_{T L O, L L O \text { or } T L O}=\frac{1}{\Delta \omega_{n}} \int_{\Delta \omega_{m n}} \frac{\gamma_{b u l k}^{2} \hbar}{3 \pi v_{p h}^{2}\left(\omega_{n}\right) v_{g}^{3}\left(\omega_{m}\right)} \omega_{n} \omega_{m}^{3} \omega_{O}\left(f_{0}\left(\omega_{m}\right)-f_{0}\left(\omega_{O}\right)\right) d \omega_{n}
\end{aligned}
$$

where $L T L$ denotes the $\mathrm{U}$ process $L+T=L, \gamma_{b u l k}$ is the bulk Grüneisen parameter, $v_{p h}\left(\omega_{n}\right)$ is the phase velocity at $\omega_{n}$ of the $n$-th band, and $r_{c}$ determines the integral surface near the first Brillouin zone(FBZ) boundary where the admissible zone-boundary phonons with frequency $\omega_{L}$ and $\omega_{T}$ reside, and it is mathematically treated in Ref. [34]. The procedure to determine $\Delta \omega_{m n}$ is further summarized in Appendix 
B in Ref. [16]: given 2 bands $n$ and $m$, searching for two frequency intervals in $\Delta \omega_{n}$ and $\Delta \omega_{m}$ such that $\Delta \omega_{m n}=\Delta \omega_{m} \cup \Delta \omega_{n}$ and energy conversation is satisfied for the phonon pairs within these two intervals (e.g. $\omega_{n}+\omega_{T}=\omega_{m}$ for $L T L$ ). Under the Matthiessen's rule, the final net band averaged inverse relaxation time $\left(\tau_{n m}^{-1}\right)_{a v g}$ is given by

$$
\left(\tau_{n m}^{-1}\right)_{a v g}=\sum_{i}\left(\tau_{n m}^{-1}\right)_{(i=L T L, T L L, \ldots)} .
$$

5. The phonon boundary scattering mechanism of nano-resonators are treated directly in phonon BTE given in Eq. (7). For Si nano-resonators, diffusive scattering is assumed at the structure boundary [36, 37, 38], i.e., the phonons $\left(v_{g} e\right)$ are reflected back at the boundary and evenly distributed across the interior solid angle of the interface. The boundary scattering re-directs the irreversible heat flows in regions near the structure boundary, and hence causing surface damping.

\subsection{Phonon perturbed mechanical equation of motion}

The dynamics of the nano-resonator with small deformation assumption is characterized by the kinematics equation $\epsilon_{i j}=\frac{\partial u_{i}}{\partial x_{j}},(i, j=1,2,3)$, equation of motion, and constitutive relation [28]:

$$
\begin{gathered}
\sigma_{i j, j}+b_{i}-\rho \ddot{u}_{i}=0, \quad(i, j=1,2,3), \\
\sigma_{i j}=\frac{\partial U_{0}}{\partial \epsilon_{i j}}-\int\left(\frac{1}{2} \hbar \omega+\frac{\hbar \omega}{1-e^{x}}\right) D(\omega) \gamma_{i j} d \omega \approx \sum_{k l} C_{i j k l}^{0} \epsilon_{k l}-\sum_{n} \int_{4 \pi} e_{n}\left(\gamma_{i j}\right)_{n} d \Omega,
\end{gathered}
$$

where $u$ is the displacement, $b$ is the body force, $C_{i j k l}^{0}$ is defined as the portion of the elastic constant that corresponds to the static lattice potential energy $U_{0}$ and the ground state phonon energy $\frac{1}{2} \hbar \omega$, which is given by

$$
C_{i j k l}^{0}=\frac{\partial^{2} U_{0}}{\partial \epsilon_{i j} \partial \epsilon_{k l}}-\int \frac{\partial\left(\frac{1}{2} \hbar \omega D(\omega) \gamma_{i j}\right)}{\partial \epsilon_{k l}} d \omega
$$

$C_{i j k l}^{0}$ can be directly obtained from the thermodynamic property calculations as described in Section 2.2. For 2-D analysis of single crystal $\mathrm{Si}, C_{i j k l}^{0}$ has only three non-zero components $C_{11}, C_{12}$ and $C_{44}$. In Eq.(15), the thermal stress part is directly expressed with the band phonon energy density $e_{n}$ and the Grüneisen parameter $\left(\gamma_{i j}\right)_{n}$.

Equations (14) and (7) form the coupled thermomechanical governing equations for nano-resonators. The damping ratio $\zeta$ is defined as

$$
\zeta=\frac{1}{2 \pi} \frac{E_{l o s t}}{E_{s t}}
$$


where $E_{l o s t}$ and $E_{s t}$ are the strain energy loss and the peak strain energy stored per unit volume per period of vibration, respectively. The quality factor is defined as $Q=\zeta^{-1}$. In general, $\zeta$ and $Q$ can be evaluated either in the time domain by tracing the system response [39], or in the frequency domain by finding the generalized eigenvalues [40,41], or by calculating the harmonic response under periodic forces [42]. In this work, the harmonic analysis approach is adopted due to its relative small computational cost.

\section{Numerical Discretization of the Governing Equations}

The boundary value problems, Eq.(14) and Eq.(7), are discretized by using FEM and FVM methods, receptively. For the dynamic analysis of the nano-structure, the weak form of Eq.(14) is obtained by using the standard Galerkin's method, given by

$$
\begin{array}{r}
\int_{V} \rho\left(\delta u_{i}\right) \ddot{u}_{i} d V+\int_{V}\left(\delta \epsilon_{i j}\right) C_{i j k l}^{0}\left(\epsilon_{k l}\right) d V=\int_{V}\left(\delta \epsilon_{i j}\right)\left(\sum_{n} \int_{4 \pi} e_{n}\left(\gamma_{i j}\right)_{n} d \Omega\right) d V \\
+\int_{V}\left(\delta u_{i}\right) b_{i} d V+\int_{A}\left(\delta u_{i}\right) f_{i} d A \quad(i, j, k, l=1,2,3),
\end{array}
$$

where $\ddot{u}$ is the acceleration, $\delta u$ and $\delta \epsilon$ are the variation of displacement and strain, respectively, $V$ and $A$ are the material volume and surface, respectively, $f$ is the surface traction, and $b$ is the body force. In the 2-D analysis, $V$ is meshed into triangular elements $V_{e}$, shown in Fig.2. The integrals in Eq. (18) are approximated and discretized into matrix form using standard shape functions, which are further assembled into the global matrix equation as

$$
\mathbf{M}_{\mathbf{u u}} \ddot{\mathbf{u}}+\mathbf{K}_{\mathbf{u u}} \mathbf{u}+\mathbf{K}_{\mathbf{u e}} \mathbf{e}=\mathbf{F}
$$

where $\mathbf{M}_{\mathbf{u u}}, \mathbf{K}_{\mathbf{u u}}$ and $\mathbf{F}$ are the mass, stiffness and force matrices, respectively, $\mathbf{K}_{\mathbf{u e}}$ is the phonon-stress coupling matrix.

The phonon BTE, Eq.(7), is discretized using the standard FVM. The solid angle $\Omega(\theta, \phi)$ is discretized into $R$ and $S$ segments in the $\theta$ (polar) and $\phi$ (azimuthal) directions, respectively, with $\Delta \Omega^{r s}$ representing a control angle, as shown in Fig.2. The finite volume spacial discretization of the phonon BTE shares the same mesh with the finite element discretization for the dynamic structural analysis described above. In FVM, each triangular element is referred to as a control volume. $e_{n P}^{r s}$ represents the phonon energy density of the $n$th energy band in control volume $P$ and along the direction of $\Delta \Omega^{r s} . \Delta A_{q}$ is the edge length. $D_{C_{q, \text { in }}}^{r s}$ and $D_{C_{q, \text { out }}}^{r s}$ are introduced as the directional weights of phonon intensities at these edges. The detailed formulation can 


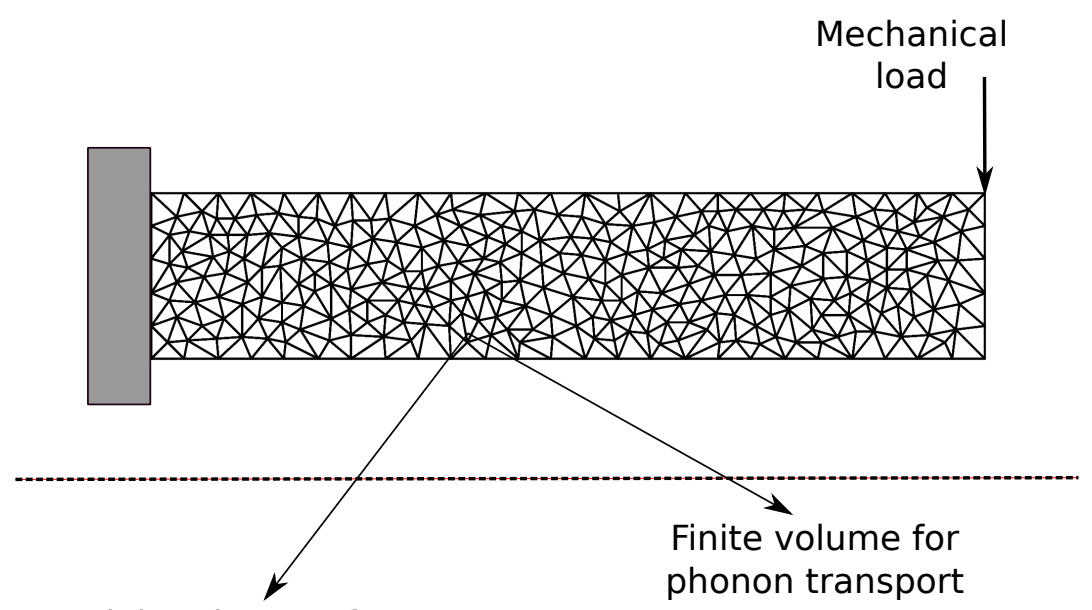

Finite element for mechanical analysis
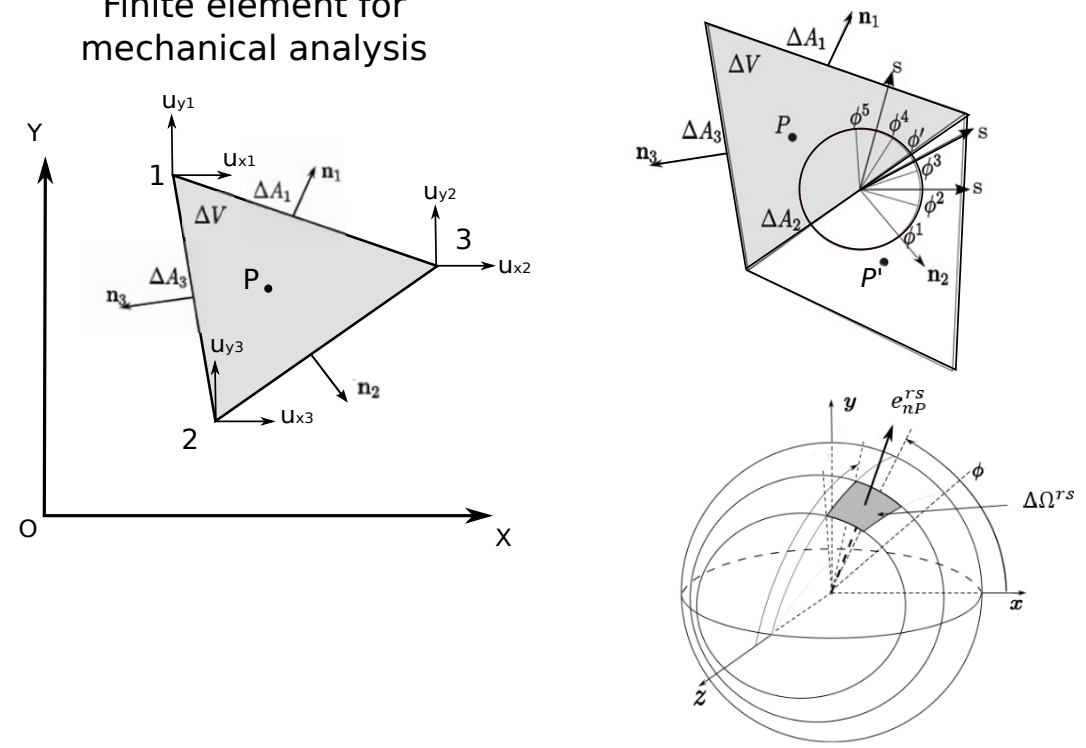

Figure 2: Top: domain discretization of a cantilever nano-resonator. Bottom left: finite element for mechanical analysis. Bottom right: finite volume for phonon transport analysis.

be found in our previous work Ref. [43]. The discretized BTE for a control volume $P$ becomes

$$
\begin{aligned}
\left(\Delta V_{P} \Delta \Omega^{r s}\right) \dot{e}_{n P}^{r s}+ & {\left[\sum_{q=1}^{3}\left(v_{g}\right)_{n} \Delta A_{q} D_{C_{q, \text { out }}}^{r s}+\left(\Delta V_{P} \Delta \Omega^{r s}-\frac{\Delta V_{P}\left(\Delta \Omega^{r s}\right)^{2}}{4 \pi}\right)\left(\tau_{n n}^{-1}\right)_{\text {avg }}\right] e_{n P}^{r s} } \\
& -\sum_{r^{\prime} s^{\prime} \neq r s}\left[\frac{\Delta V_{P} \Delta \Omega^{r s} \Delta \Omega^{r^{\prime} s^{\prime}}}{4 \pi}\left(\tau_{n n}^{-1}\right)_{\text {avg }}\right] e_{n P}^{r^{\prime} s^{\prime}}+\sum_{q=1}^{3}\left(v_{g}\right)_{n} \Delta A_{q} D_{C_{q, i n}}^{r s} e_{n P \prime}^{r s} \\
= & \sum_{m \neq n} \frac{\frac{1}{4 \pi}\left(C_{v}\right)_{n}\left(T_{n m}-T_{r e f}\right)-e_{n P}^{r s}}{\left(\tau_{n m}\right)_{a v g}} \Delta V_{P} \Delta \Omega^{r s}-\sum_{i j}\left(\phi_{i j}\right)_{n} \Delta V_{P} \Delta \Omega^{r s} \dot{\epsilon}_{i j} .
\end{aligned}
$$


If control volume $P$ has one or more edges on the structural boundary, the diffusive phonon reflection gives

$$
e_{n P}^{r s}=\frac{1}{\pi} \sum_{r s} e_{n P I}^{r s} D_{C_{q, o u t}}^{r s}
$$

Equations (20) and (21) are assembled into the global matrix equation as

$$
\mathbf{C}_{\mathrm{ee}} \dot{\mathbf{e}}+\mathbf{K}_{\mathrm{ee}} \mathbf{e}+\mathbf{C}_{\mathrm{eu}} \dot{\mathbf{u}}=\mathbf{0}
$$

where $\mathbf{C}_{\mathbf{e u}}$ is the phonon-modulation matrix. Combining Eq. (22) and Eq. (19), the matrix form of the discretized thermomechanical equation can be written as

$$
\left[\begin{array}{cc}
\mathbf{M}_{\mathrm{uu}} & \mathbf{0} \\
\mathbf{0} & 0
\end{array}\right]\left[\begin{array}{c}
\ddot{\mathbf{u}} \\
\ddot{\mathrm{e}}
\end{array}\right]+\left[\begin{array}{cc}
\mathbf{0} & \mathbf{0} \\
\mathbf{C}_{\mathrm{eu}} & \mathbf{C}_{\mathbf{e e}}
\end{array}\right]\left[\begin{array}{c}
\dot{\mathbf{u}} \\
\dot{\mathbf{e}}
\end{array}\right]+\left[\begin{array}{cc}
\mathbf{K}_{\mathrm{uu}} & \mathbf{K}_{\mathbf{u e}} \\
\mathbf{0} & \mathbf{K}_{\mathrm{ee}}
\end{array}\right]\left[\begin{array}{l}
\mathbf{u} \\
\mathbf{e}
\end{array}\right]=\left[\begin{array}{l}
\mathbf{F} \\
\mathbf{0}
\end{array}\right]
$$

Next, a harmonic analysis is performed upon the system given in Eq. (23) and damping ratio $\zeta$ is evaluated using Eq.(17). The harmonic excitation, solutions of displacement and phonon energy density are expressed in the following form,

$$
\begin{gathered}
\mathbf{F}=\mathbf{F}_{\mathbf{s}}+\mathbf{F}_{\mathbf{d}} e^{\left(i \omega_{d} t\right)} \\
\mathbf{u}=\mathbf{u}_{\mathbf{s}}+\mathbf{u}_{\mathbf{d}} e^{\left(i \omega_{d} t\right)}, \\
\mathbf{e}=\mathbf{e}_{\mathbf{s}}+\mathbf{e}_{\mathbf{d}} e^{\left(i \omega_{d} t\right)}
\end{gathered}
$$

where $\omega_{d}$ is the frequency of the harmonic excitation, and the subscripts $s$ and $d$ denote the static and dynamic components, respectively. Note that only the real parts of them have physical significance. Substitute the dynamic components into Eq.(23),

$$
-\omega_{d}^{2}\left[\begin{array}{cc}
\mathbf{M}_{\mathbf{u u}} & \mathbf{0} \\
\mathbf{0} & \mathbf{0}
\end{array}\right]\left[\begin{array}{l}
\mathbf{u}_{\mathbf{d}} \\
\mathbf{e}_{\mathbf{d}}
\end{array}\right]+i \omega_{d}\left[\begin{array}{cc}
\mathbf{0} & \mathbf{0} \\
\mathbf{C}_{\mathbf{e u}} & \mathbf{C}_{\mathbf{e e}}
\end{array}\right]\left[\begin{array}{l}
\mathbf{u}_{\mathbf{d}} \\
\mathbf{e}_{\mathbf{d}}
\end{array}\right]+\left[\begin{array}{cc}
\mathbf{K}_{\mathbf{u u}} & \mathbf{K}_{\mathbf{u e}} \\
\mathbf{0} & \mathbf{K}_{\mathbf{e e}}
\end{array}\right]\left[\begin{array}{l}
\mathbf{u}_{\mathbf{d}} \\
\mathbf{e}_{\mathbf{d}}
\end{array}\right]=\left[\begin{array}{c}
\mathbf{F}_{\mathbf{d}} \\
\mathbf{0}
\end{array}\right] .
$$

Equation (27) is solved to obtain the solution of $\mathbf{u}_{\mathbf{d}}$ and $\mathbf{e}_{\mathbf{d}}$ in complex form. For adiabatic systems, the $E_{l o s t}$ is equal to the amount of energy input per unit vibration cycle, given by

$$
E_{l o s t}=\int_{0}^{\frac{2 \pi}{\omega_{d}}} \operatorname{Re}\left(\mathbf{F}_{\mathbf{d}} e^{\left(i \omega_{d} t\right)}\right) \cdot \operatorname{Re}\left(\mathbf{u}_{\mathbf{d}} e^{\left(i \omega_{d} t\right)}\right) d t=\pi \mathbf{F}_{\mathbf{d}} \cdot \operatorname{Im}\left(\mathbf{u}_{\mathbf{d}}\right)
$$

The maximum energy stored per unit period is given by

$$
E_{s t}=\frac{1}{2} \int_{V} \operatorname{Re}\left(\sigma^{\mathbf{T}}\left(\mathbf{u}_{\mathbf{d}}\right)\right) \cdot \operatorname{Re}\left(\epsilon\left(\mathbf{u}_{\mathbf{d}}\right)\right) d V
$$

Finally, Eq.(17) is used to calculate the damping ratio $\zeta$. 


\section{Results and discussions}

\subsection{Thermodynamic properties of single crystal silicon}

In our work, the thermodynamic properties of single crystals, calculated from lattice dynamics using Tersoff potential, serves as input parameters of the coupled phonon-damping equations. Our calculations are based on the reciprocal space quasiharmonic model (QHMK) described in Section 2.1 and our previous work [28]. The results have been validated for phonon density of states $D(\omega)$, specific heat capacity $C_{v}$ and the phonon group velocity $v_{g}$ in the temperature range of $100 \mathrm{~K}$ to $500 \mathrm{~K}$ [43]. Another important phonon property, relaxation times $\left(\tau_{n m}^{-1}\right)_{\text {avg }}$ and $\left(\tau_{n n}^{-1}\right)_{\text {avg }}$, determines the extent of thermal resistance and phonon energy interaction. For validation, we compute the effective relaxation time $\left(\tau_{n}\right)_{e f f}$ as a function of $\omega$, which is defined as $\left(\tau_{n}\right)_{\text {eff }}^{-1}=\sum_{m}^{N_{b}}\left(\tau_{n m}^{-1}\right)_{\text {avg. }}$. Discretizing the acoustic phonon dispersion curve in [100] direction (see Fig. 1) into 14 bands, the $\omega$-dependent $\left(\tau_{n}\right)_{e f f}$ of $\mathrm{Si}$ at $300 \mathrm{~K}$ for both LA and TA polarizations are calculated. The results are plotted and compared with those from anharmonic lattice dynamics calculation using StillingerWeber potential [44], as shown in Fig.3. While the results are in general agreement, our results are lower in the higher frequency range, possibly due to the difference between the Tersoff and the Stillinger-Weber potentials. Nevertheless, our TA results in the higher frequency range agrees well with the empirical relation $\tau_{T, U}^{-1}=B_{T, U} \omega^{2} T e^{(\theta / 3 T)}$, where $B_{T, U}$ and $\theta$ are the calibrated scattering parameter and Debye temperature respectively [38].

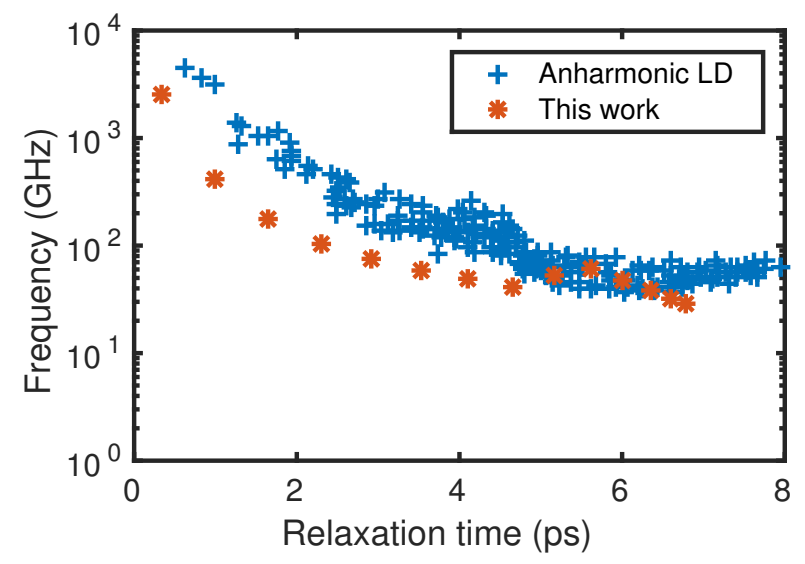

(a)

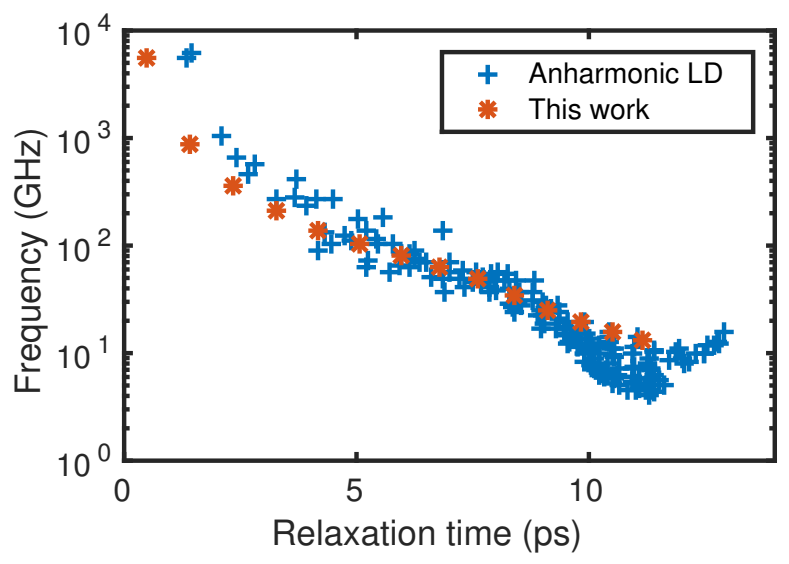

(b)

Figure 3: Effective relaxation time of Si in [100] direction at 300K: (a) LA; (b) TA. 


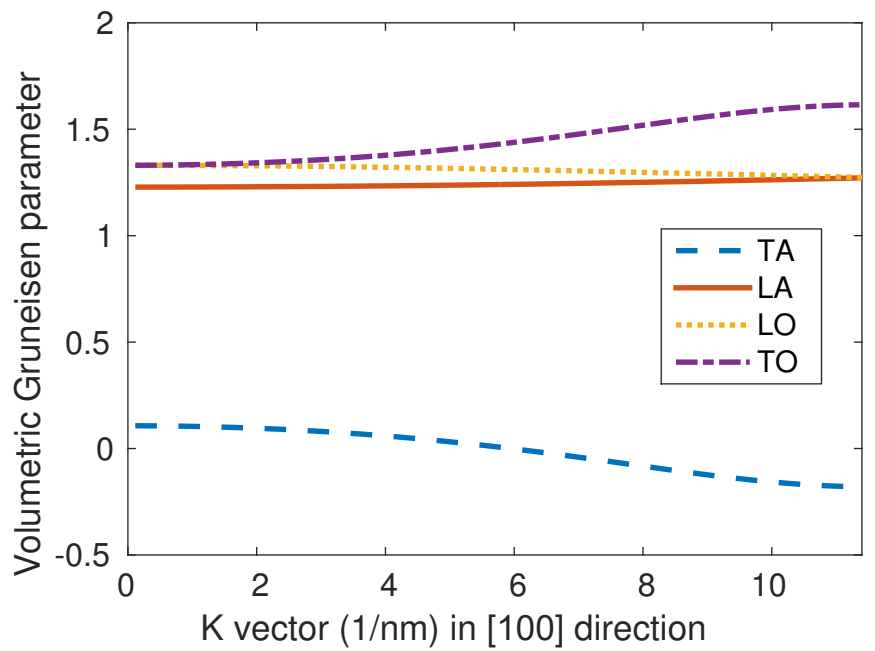

Figure 4: Dispersion of volumetric Grüneisen parameter in [100] direction

Next, as shown in Fig. 4, we compute the dispersion of volumetric Grüneisen parameter $\gamma_{V}=\frac{1}{3} \sum_{i=1}^{3} \gamma_{i i}$ in [100] direction at $300 \mathrm{~K}$. While our results are consistent with the anharmonic lattice dynamics calculation in the literature [21], significant discrepancies for TA mode are observed when they are compared to the results from ab initio calculations $[45,22]$ or experimental data [46]. In general, the calculated $\left(\gamma_{V}\right)_{T A}$ $([-0.5,-0.3])$ is higher than the experimental results $([-1.3,-0.5])$. It is known that such discrepancies lead to an overestimate of the linear thermal expansion coefficient $\alpha$ [21]. This discrepancy can be attributed to the overestimated TA frequency $\omega_{T A}$ produced by the Tersoff potential. It is worthwhile to note that the Tersoff potential, as well as others such as the Stillinger-Weber and the ED potentials, does not accurately account for all the thermodynamic properties simultaneously. While this inaccuracy can be remedied by calculating $\gamma_{i j}$ from more accurate models such as ab initio models, in this work, we intend to validate the phonon damping model against MD simulations using the same Tersoff potential. Therefore, it is expected that such inaccuracy is also presented in the MD results and does not defeat the purpose of model validation. The bulk thermodynamic properties of $\mathrm{Si}$ at $300 \mathrm{~K}$ used in the phonon damping calculations are summarized in Table 3.

It should be emphasized that the above calculations yield bulk material properties. However, finite size (surface) effects can be significant when the characteristic dimension of the nanostructures reduces to a few nanometers. For example, although the bulk elastic constants can be accurately computed by the QHMK 
Table 3: Thermodynamic properties of single crystal silicon at 300K

\begin{tabular}{ll}
\hline \hline Density & $\rho=2328.12 \mathrm{~kg} / \mathrm{m}^{3}$ \\
Bulk Grüneisen parameter & $\gamma_{b u l k}=0.5973$ \\
Phonon group velocity & $\left(v_{g}\right)_{L A}=5963.43 \mathrm{~m} / \mathrm{s}$ \\
& $\left(v_{g}\right)_{T A}=1410.07 \mathrm{~m} / \mathrm{s}$ \\
Bulk Thermal conductivity & $K=143.26 \mathrm{~W} / \mathrm{m} / \mathrm{K}$ \\
Specific Heat capacity & $\left(C_{v}\right)_{b u l k}=93.2 \times 10^{4} \mathrm{~J} / \mathrm{kg} / \mathrm{K}$ \\
\hline
\end{tabular}

model in the temperature range of $100 \mathrm{~K}$ to $800 \mathrm{~K}$, these bulk values overestimate the stiffness of the nanoresonators with cross sectional area smaller than $10 \mathrm{~nm}^{2}$ in comparison with MD results [47]. To eliminate this inaccuracy, finite size elastic constants are determined and validated against MD results to ensure consistent and accurate mechanical response and strain energy. The procedure is detailed in Appendix A. In general, finite size effects exist in all thermodynamic properties. However, due to the lack of efficient theoretical models to incorporate all of such effects, we use bulk values of $D(\omega), C_{v}, v_{g},\left(\tau_{n m}^{-1}\right)_{a v g},\left(\tau_{n n}^{-1}\right)_{a v g}$ and $\gamma_{i j}(\omega)$ in the phonon BTE, and finite size elastic constants $C_{11}, C_{12}$ and $C_{44}$ in the mechanical equation. In the following discussion of numerical results for different damping cases, the finite size effects are further discussed and the size limit of the bulk property approximation is also shown for individual damping cases.

\subsection{Damping ratio of single crystal Si nano-resonators}

In this section, the damping ratio of 3 different types of Si nano-resonators subject to harmonic loading is calculated. The results obtained by using the phonon damping model are compared to those from MD simulations. The MD simulations were performed using the LAMMPS code [14]. In all MD simulations, the structure was equilibrated at $300 \mathrm{~K}$ using a Nose-Hoover thermostat with a time constant of $0.1 \mathrm{ps}$. After equilibration of the structure for $2 \mathrm{~ns}$, a harmonic force was applied to the atoms within one unit cell distance from the right end. The system was further evolved as a NVE ensemble for a simulation time of 200 cycles. A time step of $1 \mathrm{fs}$ was used for all simulations. The energy lost per unit period $E_{\text {lost }}$ is equal to the external work done to maintain harmonic vibration in one period, and it follows that the external work equals to the increase in the system internal energy $\Delta U$ from the law of thermodynamics. The time history of internal energy is output from MD simulations and $\Delta U$ is measured. The stored strain energy $E_{s t}$ is taken as the same 
value calculated from Eq. (29) in the theoretical model. The equivalence of $E_{s t}$ in both MD and our model is described in Appendix A. The damping ratio $\zeta$ is then calculated using Eq. (17).

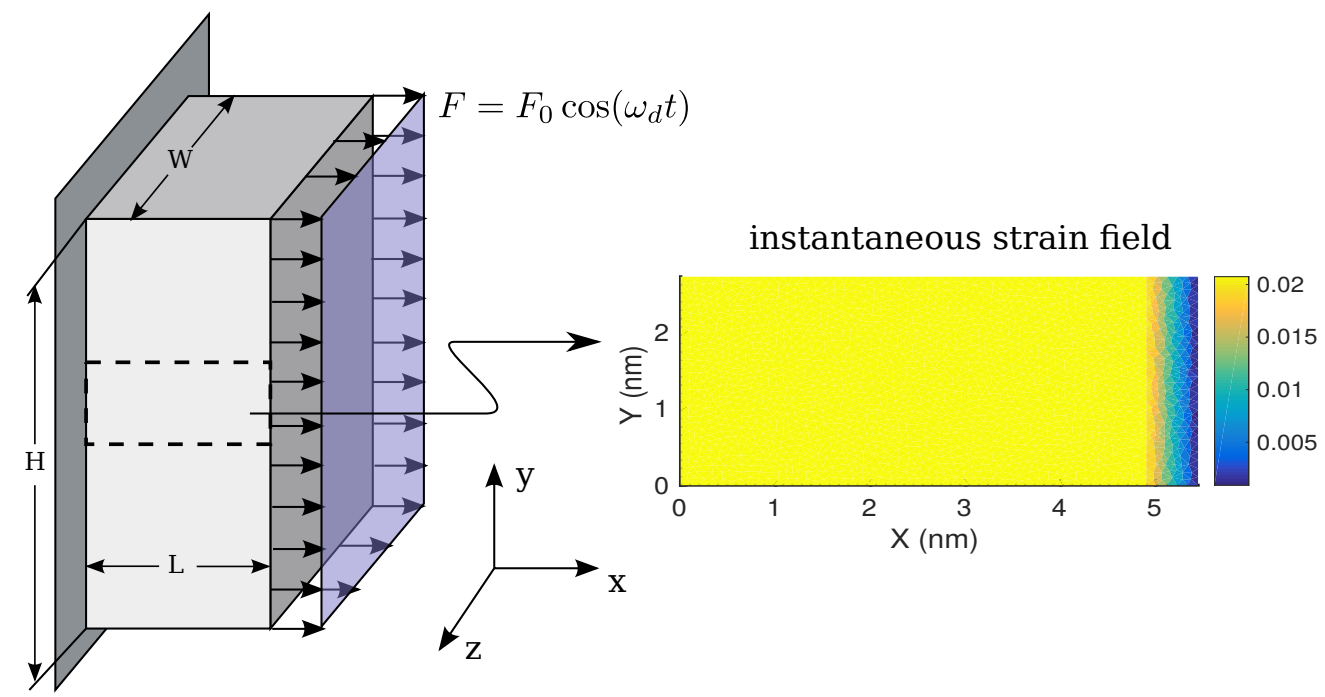

Figure 5: Phonon damping analysis of the "Block" resonator case: schematic (left) and an instantaneous profile of $\epsilon_{x x}$ under the harmonic load (right).

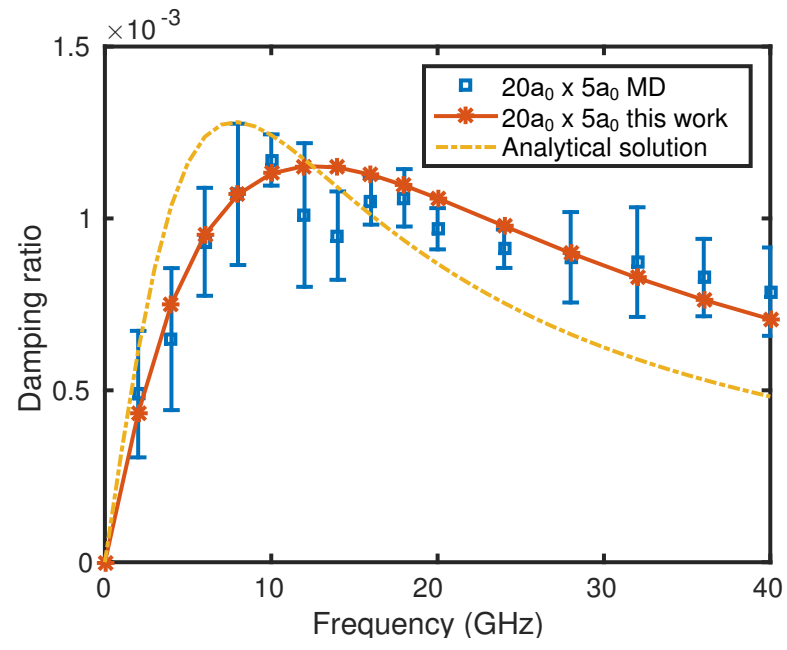

(a)

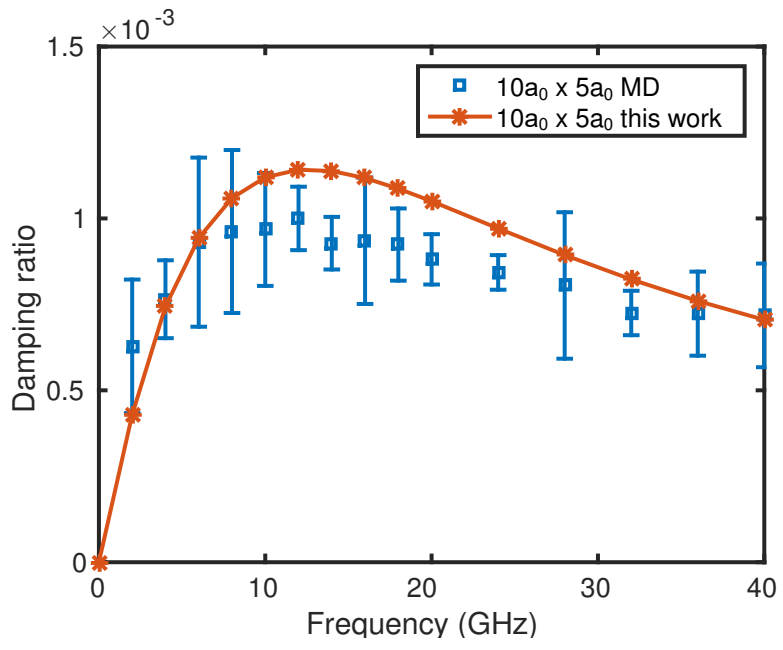

(b)

Figure 6: Damping of "Block" resonators: damping ratio $\zeta$ verses frequency $\omega_{d}$ : (a) length $=20 a_{0}$, (b) length $=10 a_{0}$.

As shown in Fig. 5, the first case we consider is a Si nano-resonator with width (W) and height $(\mathrm{H})$ 
much larger than length (L) and subject to a harmonic load in the x-direction. This case is referred as "Block" resonator case in the following. In the quasi-continuum phonon damping analysis, the resonator is represented as a 2-D plane strain model with periodic boundary conditions applied on the top and bottom edges. The resonator has a length ranging from $L=5.4464 \mathrm{~nm}\left(10 a_{0}\right)$ to $L=10.8928 \mathrm{~nm}\left(20 a_{0}\right)$ and a height of $H=2.7232 \mathrm{~nm}\left(5 a_{0}\right)$. In the MD simulations, a square cross section of $2.7232 \mathrm{~nm} \times 2.7232 \mathrm{~nm}\left(5 a_{0} \times 5 a_{0}\right)$ with periodic boundary in both $y$ - and z-directions is defined for the resonator. In the harmonic force, $\omega_{d}$ is set to vary from $2 \mathrm{GHz}$ to $40 \mathrm{GHz}$, and the amplitude $F_{0}$ is set such that $\epsilon_{\max }=0.02$. The phonon damping in this case can be characterized as primarily Akhiezer damping since the thermoelastic damping is minimal in the presence of a nearly uniform instantaneous strain field as indicated in Fig. 5 (right). Furthermore, the surface effects is largely eliminated by the small surface-volume ratio. Figure 6(a) shows a comparison of the damping ratio $\zeta$ calculated from the quasi-continuum model and MD simulation for block length $L=10.8928$ $\mathrm{nm}\left(20 a_{0}\right)$. The results are generally in accordance with each other and clearly show the Lorentzian behavior: $\zeta$ first increases up to $1.14 \times 10^{-3}$ at around $12 \sim 13 \mathrm{GHz}$ and then decreases as $\omega_{d}$ becomes larger. This damping behavior has also been demonstrated by a separate study recently [48]. In the light of pure Akhiezer damping, an analytical relation between $\zeta$ and $\omega_{d}$ was proposed in Ref. [19] based on an analytical solution of the single mode Boltzmann transport equation, which is given by

$$
\zeta=\frac{1}{2 \pi} \frac{\gamma_{e f f}^{2}\left(C_{v}\right)_{b u l k} T}{\rho\left(v_{g}\right)_{b u l k}^{2}} \frac{\omega_{d} \tau_{p h-p h}}{1+\left(\omega_{d} \tau_{p h-p h}\right)^{2}},
$$

where $\gamma_{e f f}$ is the effective Grüneisen parameter, (see Eq. (6) in Ref. [19]), $\tau_{p h-p h}$ is a metrics for assessing the peak Akhiezer damping taking place at $\omega_{d} \tau_{p h-p h}=1$, and was found to be of the same magnitude as the effective phonon relaxation time (tens of $p s$ for $\mathrm{Si}$ ). For a comparison, by taking $\gamma_{\text {eff }}=\gamma_{b u l k}$, and the effective phonon relaxation time $\tau_{p h-p h}$ from the kinetic theory as

$$
\tau_{p h-p h}=\frac{3 K}{\left(C_{v}\right)_{b u l k}\left(v_{g}\right)_{b u l k}^{2}}
$$

where $K$ is the bulk thermal conductivity. $\zeta$ from Eq. (30) is calculated and plotted in Fig. 6(a). While, from this simple model, the peak $\zeta$ occurs at a lower frequency, a similar Lorentzian trend is observed. Since the Akhiezer damping is due to phonon redistribution across the phonon modes (bands) only, it is a local phenomenon and a change in block length $L$ is expected to have a small effect on $\zeta$. Figure 6(b) shows the results of $\zeta$ for a smaller block length $L=5.4464 \mathrm{~nm}\left(10 a_{0}\right)$. While the $\zeta$ calculated from the quasi-continuum model shows little difference compared to that of $L=10.8928 \mathrm{~nm}\left(20 a_{0}\right)$, the MD result becomes smaller by $10 \%$. This is due to the fact that as the block length reduces, the surface to volume ratio 
increases and the phonon properties of the system change from their bulk values. It has been observed in MD simulations [14] that the presence of surface in nano-structures significantly reduces the effective phonon phonon relaxation time in low frequency longitudinal range. While the quasi-continuum model uses the bulk dispersion relation, density of states, phonon relaxation and Grüneisen parameters in the calculation, the finite size effect on the material properties directly manifests in the MD results. The result indicates that, for the "Block" resonators, the lower size limit of the quasi-continuum model is around $10 \mathrm{~nm}$.

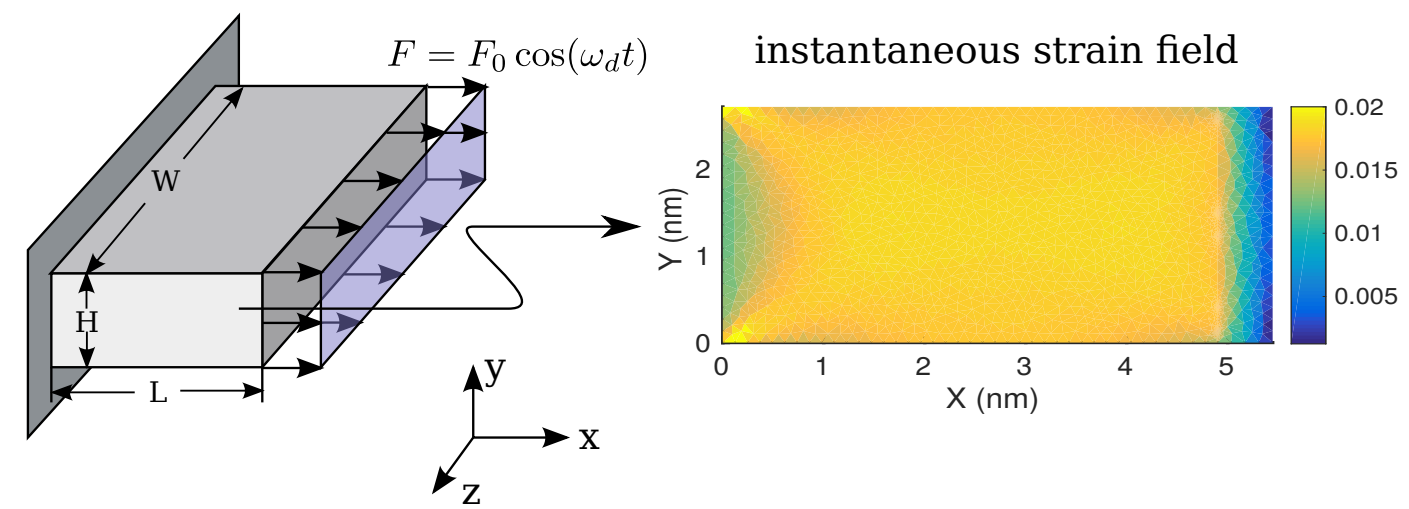

Figure 7: Phonon damping analysis of "Plate" resonator case: schematic (left) and an instantaneous profile of $\epsilon_{x x}$ under the harmonic load (right).

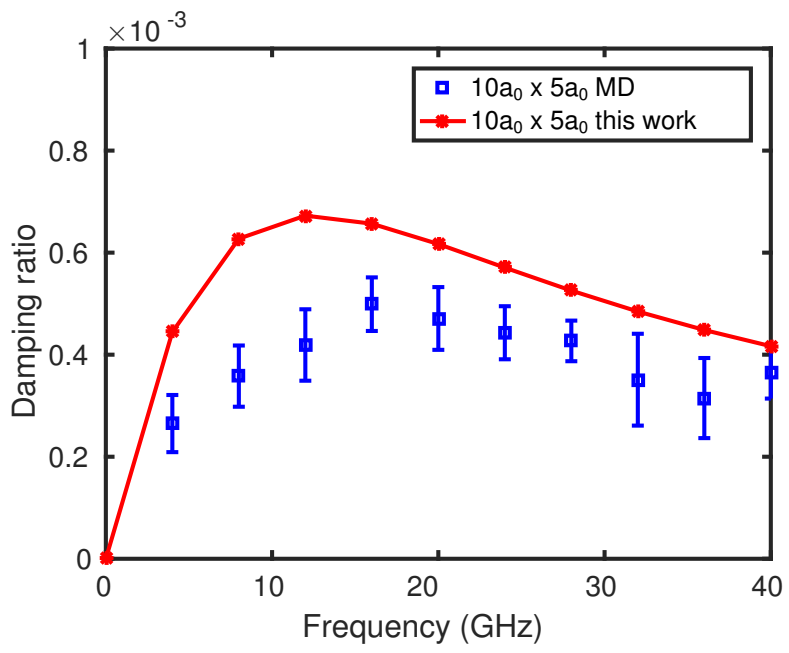

(a)

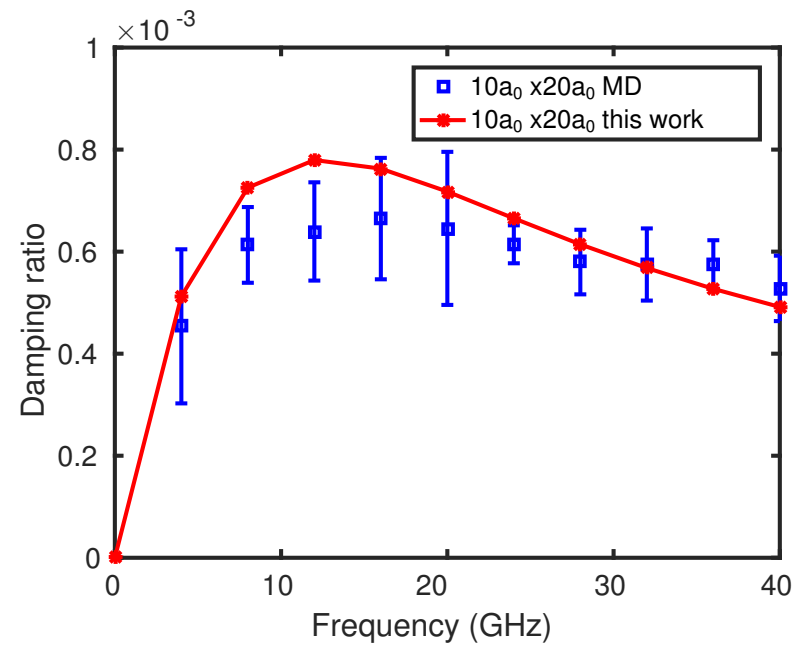

(b)

Figure 8: Damping of "Plate" resonators: damping ratio $\zeta$ as a function of frequency $\omega_{d}$ : (a) length $\times$ height= $10 a_{0} \times 5 a_{0}$, (b) length $\times$ height $=10 a_{0} \times 20 a_{0}$. 
The second simulation case is the longitudinal damping of a plate with finite height in the y-direction. This case is referred to as "Plate" resonator case. The geometry is depicted in Fig. 7, with the block length set to be $L=5.4464 \mathrm{~nm}\left(10 a_{0}\right)$, finite height ranging from $H=2.7232 \mathrm{~nm}\left(5 a_{0}\right)$ to $H=10.8928 \mathrm{~nm}$ $\left(20 a_{0}\right)$. In MD simulations, the width in the z-direction is set to be $W=2.7232 \mathrm{~nm}\left(5 a_{0}\right)$ with periodic boundary condition in the z-direction. The harmonic force $\mathbf{F}$ acts along the x-direction with $\omega_{d}$ ranging from $2 \mathrm{GHz}$ to $40 \mathrm{GHz}$, and $F_{0}$ set such that $\epsilon_{\max }=0.02$. Figure 8 shows the damping ratio $\zeta$ calculated from the quasi-continuum model and the MD simulations. Compared to the results from the "Block" resonator case, the damping ratio $\zeta$ is found to be slightly smaller, with the peak residing between $0.7 \times 10^{-3}$ and $0.8 \times 10^{-3}$. To understand this behavior, we first inspect the strain filed of $\epsilon_{x x}$ shown in Fig. 7 (right). Contrary to the "Block" resonator case shown in Fig. 5 (right), the strain field is no longer uniform in the structure due to the effect of top and bottom surfaces, which means spatial transport of phonons takes place in addition to the intramode transport. For the phonons out of equilibrium, there is competition between the thermoelastic, Akhiezer and surface boundary damping mechanisms. A portion of these phonons flows between different modes as intra-mode heat flow which leads to the Akhiezer damping, another portion of the phonons transports spatially in order to reach a new local equilibrium, and some other phonons are scattered by the structural boundary, causing combined thermoelastic and surface damping. A qualitative estimate on the relative contributions of the combined thermoelastic and surface mechanisms can be made by using Zener's linear thermoelastic damping theory [5] given by,

$$
\zeta_{\text {ted }}=\frac{1}{2 \pi}\left(\frac{\alpha^{2} E T}{\left(C_{v}\right)_{\text {bulk }}}\right)\left(\frac{\omega_{d} \tau_{\text {th }}}{1+\left(\omega_{d} \tau_{\text {th }}\right)^{2}}\right)
$$

where $E$ is the effective Young's modulus and $\tau_{t h}$ is thermal diffusion time. In this case, $\tau_{t h}$ is primarily influenced by the phonon boundary scattering time, given by $\tau_{t h} \approx \tau_{b}=\frac{H}{v_{g}}$, where $H$ is the characteristic length of the structure. For the "Plate" resonators, it can be shown that $\omega_{d} \tau_{t h}$ is on the order of $10^{-1} \sim 10^{-2}$ and $\frac{\omega_{d} \tau_{t h}}{1+\left(\omega_{d} \tau_{t h}\right)^{2}} \approx \omega_{d} \tau_{t h}$. The term $\frac{\alpha^{2} E T}{\left(C_{v}\right)_{b u l k}}$ in Eq. (32) is calculated to be on the same order of the term $\frac{\gamma_{e f f}^{2}\left(C_{v}\right)_{b u l k} T}{\rho\left(v_{g}\right)_{b u l k}^{2}}$ in Eq. (30). Therefore, when a significant portion of the phonons undergo spatial transport instead of intra-mode flow, the contribution of the combined thermoelastic and surface damping is of $2 \sim 3$ orders of magnitude lower than the Akhiezer damping, which in effect lowers the total damping ratio $\zeta$.

A comparison between Fig. 8(a) and (b) shows that, for the same vibration frequency, the "Plate" resonator with larger height $\left(10 a_{0} \times 20 a_{0}\right)$ has a higher damping ratio $\zeta$ as predicted by both MD simulation and the quasi-continuum model. Using the above reasoning, the strength of combined thermoelastic and surface damping for those phonons undergo spatial transport is approximately proportional to $\omega_{d} \tau_{t h} \approx \omega_{d} \frac{H}{v_{g}}$, so 
that increase in the height $H$ leads to increase in the damping strength. Since phonon intra-mode flow and Akhizier damping are local phenomena and depends mainly on the frequency and phonon-phonon scattering $\left(\frac{\omega_{d} \tau_{p h-p h}}{1+\left(\omega_{d} \tau_{p h-p h}\right)^{2}}\right)$, the damping strength is less influenced by the structure size and boundary. Therefore, the total damping ratio $\zeta$ for larger "Plate' resonators is slightly higher.

Comparison between the quasi-continuum and MD results shows that for "Plate" resonators the quasicontinuum model overpredicts the damping ratio $\zeta$. It is observed that the overprediction becomes less as the size of the resonator increases. Therefore, it is expected that discrepancy between the quasi-continuum and MD results is largely due to the size effects. Similar to the "Block" resonators, for "Plate" resonators with characteristic lengths smaller than $10 \mathrm{~nm}$, more accurate treatment of the size effects on the phonon properties used in the quasi-continuum model is necessary.

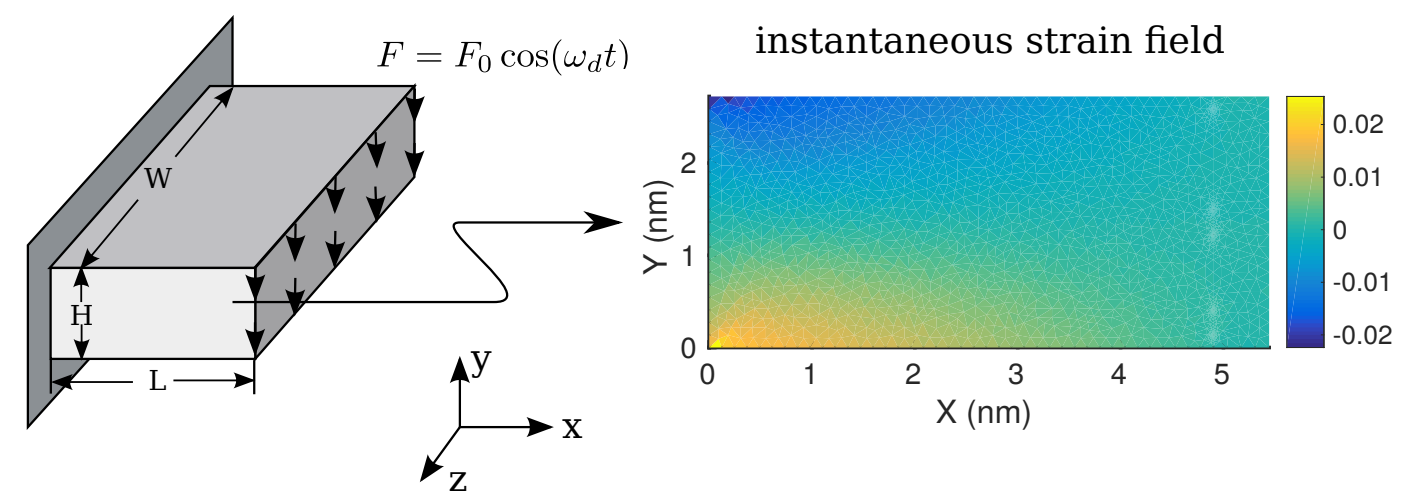

Figure 9: Phonon damping analysis of "Beam" resonator case: schematic (left) and an instantaneous profile of $\epsilon_{x x}$ under the harmonic load (right).

The third simulation case is the damping of a plate in transverse vibration mode. This case is referred to as the "Beam" resonator case. The geometry is depicted in Fig. 9. The length is set from $L=5.4464$ $\mathrm{nm}\left(10 a_{0}\right)$ to $21.7856 \mathrm{~nm}\left(40 a_{0}\right)$ with aspect ratio fixed at 2 . In MD simulations, the width $(\mathrm{W})$ is set to be $W=2.7232 \mathrm{~nm}\left(5 a_{0}\right)$ with periodic boundary condition in the z-direction. The harmonic force $\mathbf{F}$ acts along the y-direction with $\omega_{d}$ ranging from $2 \mathrm{GHz}$ to $20 \mathrm{GHz}$, and $F_{0}$ set such that $\epsilon_{\max }=0.02$. Figure 10 shows the damping ratio $\zeta$ calculated from the quasi-continuum model and the MD simulations. For 3 different sizes, it is observed that, $\zeta$ follows a near-linear relation with $\omega_{d}$. For the same structural dimensions, $\zeta$ of transverse vibration is generally smaller than that of the longitudinal vibration in the "Plate" resonator case. This behavior was also observed in an MD study of Cooper and Nickel nano-resonators [49]. To elucidate the behavior of $\zeta$, an instantaneous strain profile in the "Beam" resonator is shown in Fig. 9 (right), 


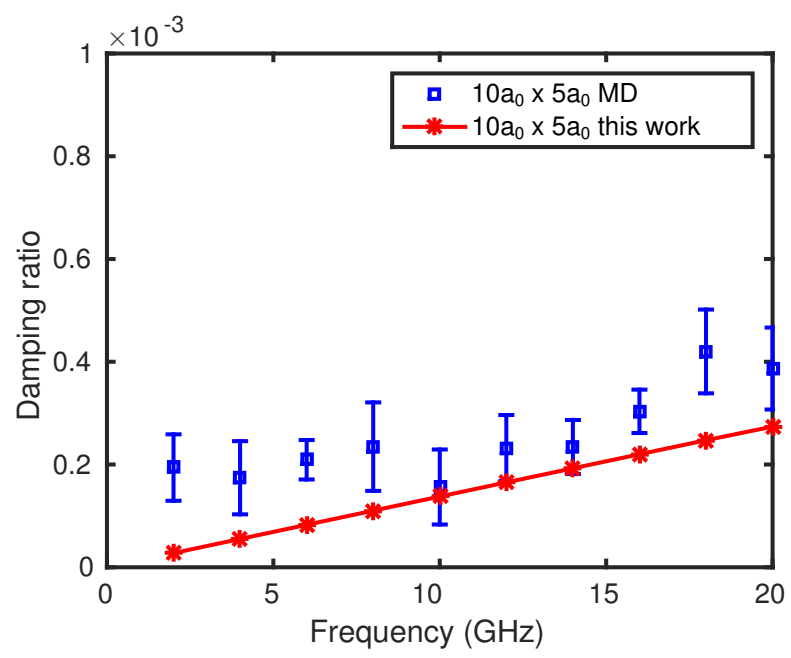

(a)

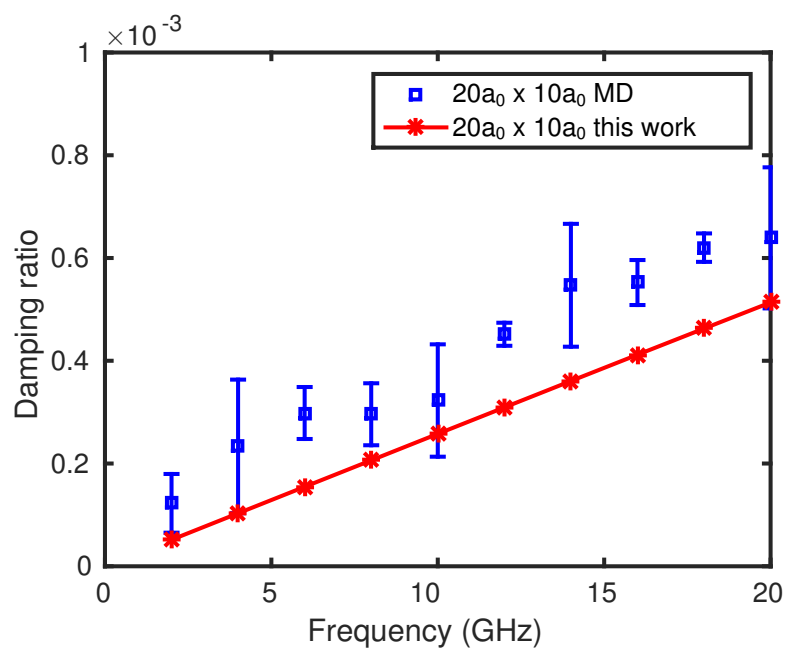

(b)

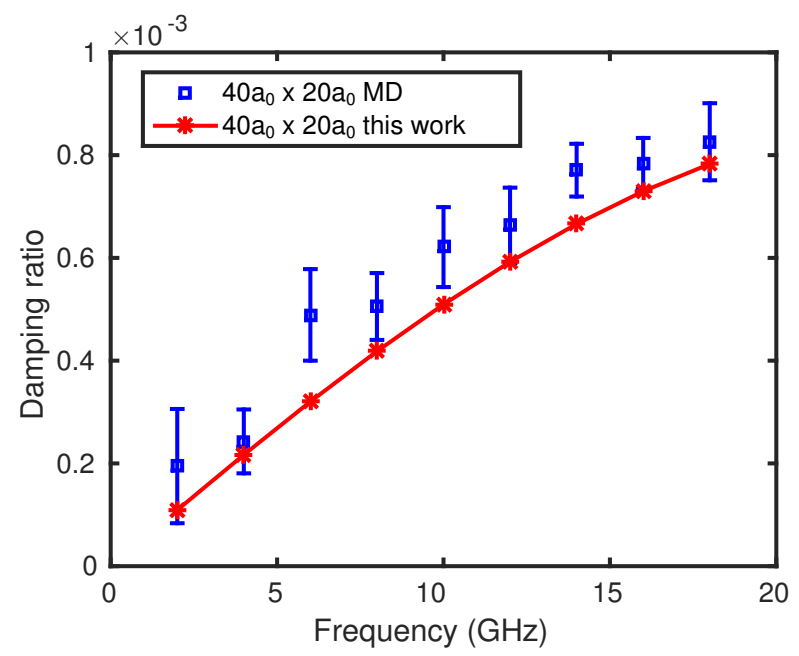

(c)

Figure 10: Damping of "Beam" resonators: damping ratio $\zeta$ as a function of frequency $\omega_{d}$ : (a) length $\times$ height $=10 a_{0} \times 5 a_{0}$, (b) length $\times$ height $=20 a_{0} \times 10 a_{0}$, (c) length $\times$ height $=40 a_{0} \times 20 a_{0}$.

which indicates clear strain gradients along both lateral and longitudinal directions. The varying strain field creates spatial thermal gradient between the dense and sparse phonon energy regions, causing a dominant spatial phonon transport. For the nano-resonators, it is clear that the phonon relaxation is mainly achieved by boundary scattering. From Zener's equation (Eq. (32)), the combined thermoelastic and surface damping ratio, $\zeta$, increases proportionally with $\frac{\omega_{d} \tau_{b}}{1+\left(\omega_{d} \tau_{b}\right)^{2}} \approx \omega_{d} \tau_{b} \approx \omega_{d} \frac{H}{v_{g}}$. The lower magnitude of $\zeta$ compared to the "Block" and "Plate" resonator cases is due to the small magnitude of $\omega_{d} \frac{H}{v_{g}}\left(10^{-2}\right.$ to $\left.10^{-1}\right)$. It should be noted 
that the above result implies the weak influence of the Akhizier effect (or phonon intra-band transport and scattering) when there are strong spatial thermal energy gradients and significant boundary scattering.

Figure 10 also indicates that, for a given vibration frequency, larger structure size gives higher damping ratio $\zeta$. This can be readily understood that increase in the height $H$ leads to increase in $\tau_{b}$ and $\zeta$. It is observed that the slopes of $\zeta$ are generally proportional to the height $H$, as shown in Table 4 . It should be noted that the linear trend in Fig. 10(c) flattens towards the right end. This is due to the fact that the linear relation is only valid when $\frac{\omega_{d} \tau_{b}}{1+\left(\omega_{d} \tau_{b}\right)^{2}} \approx \omega_{d} \tau_{b}$. For larger structures, assuming boundary scattering still dominates, the slope of $\zeta$ is expected to flatten out and approach to a peak value.

Table 4: Slope of $\zeta$

\begin{tabular}{|l|l|l|}
\hline Structure size & Model & Slope \\
\hline \multirow{2}{*}{$10 a_{0} \times 5 a_{0}$} & Quasi-continuum & $1.370 \times 10^{-5}$ \\
\cline { 2 - 3 } & MD & $1.333 \times 10^{-5}$ \\
\hline \multirow{2}{*}{$20 a_{0} \times 10 a_{0}$} & Quasi-continuum & $2.568 \times 10^{-5}$ \\
\cline { 2 - 3 } & MD & $2.702 \times 10^{-5}$ \\
\hline \multirow{2}{*}{$40 a_{0} \times 20 a_{0}$} & Quasi-continuum & $4.248 \times 10^{-5}$ \\
\cline { 2 - 3 } & MD & $4.226 \times 10^{-5}$ \\
\hline
\end{tabular}

In the "Beam" resonator case, it should also be noted that the group velocity plays an important role in the thermoelastic and surface damping. Such role of group velocity on flexural damping is also observed in Cooper and Nickel nano resonators [49]. It is well known that phonon group velocities reduce due to phonon confinement as the structural size becomes smaller. They can be approximately calculated by first calculating the vibrational modes of a nanostructure [50], and then numerically differentiating the modal frequency with respect to wave vectors. Therefore, the group velocities depend on finite size elastic constants and structure boundary. In the "Beam" resonator case, we have incorporated this phonon confinement effect using a first order correction $v_{g}^{\prime}=\sqrt{\frac{C_{i j}}{C_{i j}^{\prime}}} v_{g}$, where / denotes finite size group velocity values. The boundary influence on lower frequency phonon group velocities is ignored due to its negligible contribution. Comparing the quasicontinuum and MD results shown in Fig. 10, while both results show the same size effect, the two results match better for the larger resonators. For smaller resonators, the quasi-continuum model under-predicts $\zeta$, indicating again the size limit of the quasi-continuum model is about $10 \mathrm{~nm}$. 


\section{Conclusions}

In this paper, a coupled quasi-continuum thermo-mechanical model for intrinsic damping analysis of nanoscale resonators is presented. The model is based on the theories of phonon modulation, continuum elasticity and the particle picture of phonon thermal transport. We also present the numerical implementation of the model using combined FEM and FVM methods for calculating the damping ratio and quality factor of single crystal silicon nano-resonators under forced vibration. The numerical results are compared with results obtained from MD simulations. The numerical results show that, for nano-resonators subject to longitudinal vibration the effect of surface-volume ratio on the damping behavior is significant. For structures with minimum surface-volume ratio, the damping is primarily Akhiezer damping. The damping ratio exhibits a Lorentzian behavior as a function of vibration frequency. For nano-resonators with significant surface-volume ratio, the modulated phonons undergo Akhiezer, thermoelastic and surface mechanisms simultaneously to reach thermal equilibrium. In this case, the damping ratio still shows the Lorentzian behavior but with a reduced magnitude. For nano-resonators subject to flexural vibration and having significant surface-volume ratio, the strain profile exhibits strong spatial gradient during the vibration. In this case, the thermoelastic and surface damping are dominant and damping ratio varies nearly linearly with the frequency. Comparison of the results from the quasi-continuum model and MD simulations shows that the lower size limit of the quasi-continuum model is around $10 \mathrm{~nm}$. Below this size, the finite size effects of the elastic and phonon properties of the nano-resonators must be incorporated in the quasi-continuum model for accurate prediction of the damping ratio. This aspect of the quasi-continuum model will be explored in our future work.

\section{Acknowledgment}

We gratefully acknowledge support by the National Science Foundation under Grant CBET-0955096.

\section{Appendix A: Finite size elastic constants computation and mechanical response comparisons between MD and quasi-continuum model}

For nano-structures with characteristic length less than $10 \mathrm{~nm}$, size effect can significantly alter the elastic properties away from their bulk value due to diminished atom bonds at the surface. In this appendix, we deduce the finite-size elastic constants by comparing the mechanical responses from the continuum model and MD simulation. The finite-size elastic constants are then used in the quasi-continuum model to evaluate 
the stored vibrational strain energy $E_{s t}$ in Eq.(17), which are used in both the quasi-continuum model and MD simulation to calculate damping ratio $\zeta$.

The "Block" resonator shown in Fig. 6 has infinitely large width and height. Therefore, it is essentially a 1-D deformation problem and only $C_{11}$ is significant in the analysis. We determine the finite size $C_{11}$ by performing a MD simulation in which the static force $F_{0}$ is applied on the right end unit cell atoms in the longitudinal direction. The time-averaged center-of-mass x-directional displacement $u_{x}$ of the right end unit cell atoms is computed and linearly fitted against force of different magnitudes. The gradient $k$ of the resultant force-displacement curve gives the axial stiffness of the structure: $C_{11}=\frac{k L}{A}$, where $A$ is the cross sectional area and $L$ is measured from the left fixed end to the center-of-mass of the right end unit cell atoms. After the finite size $C_{11}$ is obtained, it is used in a continuum finite element mechanical analysis equivalent to the MD simulation to verify that both results are the same (difference less than $2 \%$ ). Then, the finite size $C_{11}$ is incorporated in the quasi-continuum model and $E_{s t}$ is calculated using Eq. (29) in the damping analysis. As shown in Fig. 8, the "Plate" resonator with infinitely large width but finite height can be approximated as a plane-strain structure. The effective stiffness along the x-direction is significant in the analysis and $C_{11}$ and $C_{12}$ should be corrected for finite size effect. Similar to the "Block" resonator, the finite size $C_{11}$ and $C_{12}$ are determined by matching the continuum mechanical analysis results with the MD simulation results for harmonic axial loads. Finally, the "Beam" resonator shown in Fig. 10 is also a plane strain structure, but subject to bending deformation. With an aspect ratio of 2 , the shear train is also significant. Therefore, the finite size $C_{11}, C_{12}$ and $C_{44}$ are determined. Under harmonic transverse loads, similar to the previous two cases, the finite size elastic constants for the "Beam" resonator are determined by matching the continuum mechanical analysis results with the MD simulation results.

The finite size elastic constants for the three types of Si nano-resonators determined for the damping analysis are summarized in Table 5. It is clear that the finite size effect on the elastic constants becomes large when the characteristic size of the nano-structures reduces to below $10 \mathrm{~nm}$. Figure 11 shows the comparison of the mechanical responses obtained from the MD simulation and the quasi-continuum model using the corrected elastic constants. 
Table 5: Finite size elastic constants of Si nanostructures

\begin{tabular}{|l||l|l|l|}
\hline Elastic Constant $(\mathrm{GPa})$ & $C_{11}$ & $C_{12}$ & $C_{44}$ \\
\hline Bulk MD $^{[28]}$ & 138.4 & 73.8 & 66.8 \\
\hline Bulk QHKM $^{[28]}$ & 137.3 & 73.1 & 66.2 \\
\hline \hline Block resonator $\left(10 a_{0} \times 5 a_{0}\right)$ & 130.6 & N/A & N/A \\
\hline Block resonator $\left(20 a_{0} \times 5 a_{0}\right)$ & 134.9 & N/A & N/A \\
\hline Plate resonator $\left(10 a_{0} \times 5 a_{0}\right)$ & 128.6 & 63.8 & N/A \\
\hline Plate resonator $\left(10 a_{0} \times 20 a_{0}\right)$ & 130.4 & 67.3 & N/A \\
\hline Beam resonator $\left(10 a_{0} \times 5 a_{0}\right)$ & 128.6 & 63.8 & 62.7 \\
\hline Beam resonator $\left(20 a_{0} \times 10 a_{0}\right)$ & 133.9 & 65.5 & 64.2 \\
\hline Beam resonator $\left(40 a_{0} \times 20 a_{0}\right)$ & 136.9 & 67.3 & 65.9 \\
\hline
\end{tabular}

\section{References}

[1] J.H. Harlow. Electric Power Transformer Engineering. The Electric Power Engineering Hbk, Second Edition. CRC Press, 2004.

[2] J. Lee, S. Lee, C. Yao, and W. Fang. Comments on the size effect on the microcantilever quality factor in free air space. Journal of Micromechanics and Microengineering, 17(1):139, 2007.

[3] C. Zener. Internal friction in solids. I. theory of internal friction in reeds. Physical Review, 52(3):230235, August 1937.

[4] D. Garrett, W. Ignacio, W. Katharina, R. Michael, and A. Markus. Phonon-tunnelling dissipation in mechanical resonators. Nature Communications, 2:231, January 2011.

[5] C. Zener. Internal friction in solids II. general theory of thermoelastic internal friction. Physical Review B, 53(1):90-99, January 1938.

[6] A. Akhiezer. On the absorption of sound in solids. Journal of Physics (Moscow), 1:227, 1939.

[7] L. G. Villanueva and S. Schmid. Evidence of surface loss as ubiquitous limiting damping mechanism in sin micro- and nanomechanical resonators. Phys. Rev. Lett., 113:227201, Nov 2014. 


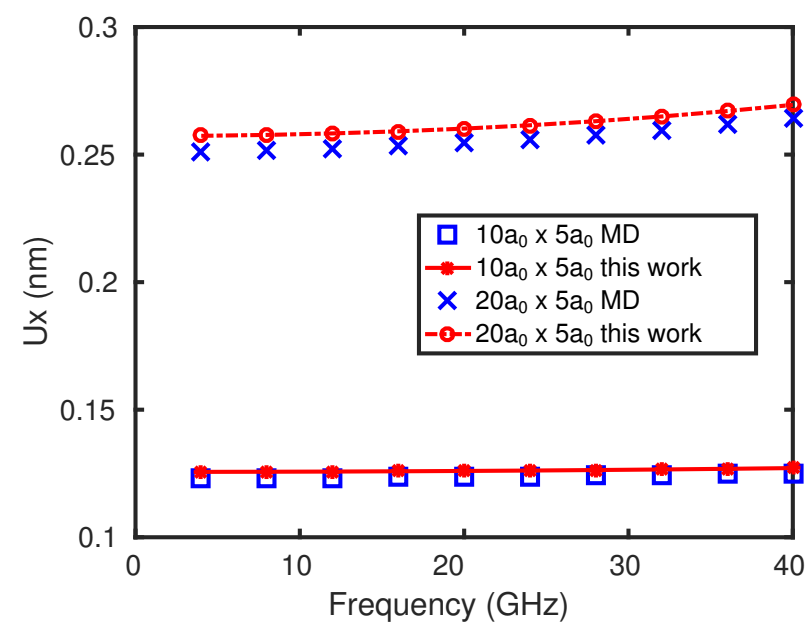

(a)

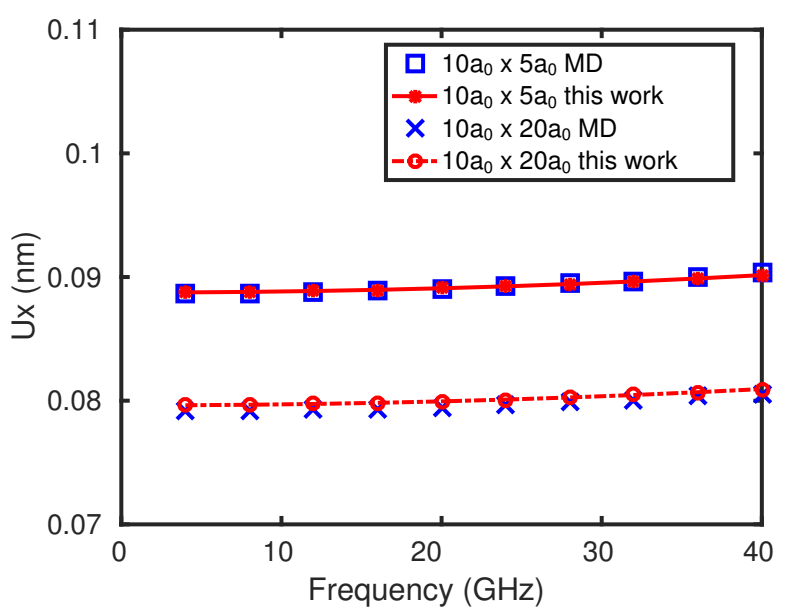

(b)

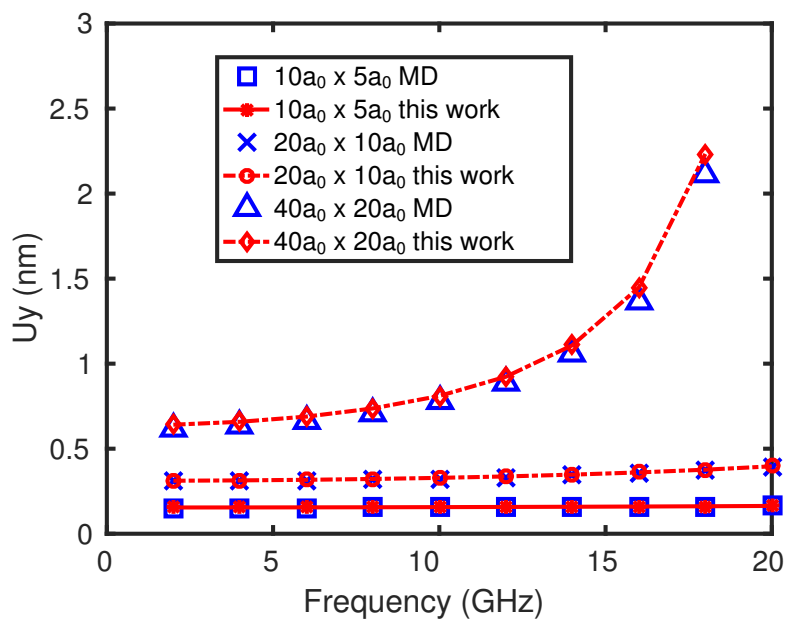

(c)

Figure 11: Comparison of vibrational displacements obtained from the MD simulation and the quasicontinuum model using the corrected elastic constants: (a) "Block" resonator, (b) "Plate" resonator, (c) "Beam" resonator.

[8] R. Lifshitz and M. L. Roukes. Thermoelastic damping in micro- and nanomechanical systems. Physical Review B, 61(8):5600-5609, February 2000.

[9] H. W. Lord and Y. Shulman. A generalized dynamical theory of thermoelasticity. Journal of the Mechanics and Physics of Solids, 15(5):299-309, 1967.

[10] Z. F. Khisaeva and M. Ostoja-Starzewski. Thermoelastic damping in nanomechanical resonators with 
finite wave speeds. Journal of Thermal Stresses, 29(3):201-216, March 2006.

[11] D. S. Chandrasekharaiah. Thermoelasticity with second sound: a review. Applied Mechanics Reviews, 39, 1986.

[12] I. Cientifieas, R. Nava, M. Vecchi, J. Romero, and B. Fernández. Akhiezer damping and the thermal conductivity of pure and impure dielectrics. Physical Review B, 14(2):800-807, July 1976.

[13] M. F. Lewis. Attenuation of longitudinal ultrasonic waves in insulators at room temperature. Journal of Acoustical Society of America, 43:4, 1968.

[14] K. Kunal and N. R. Aluru. Akhiezer damping in nanostructures. Physical Review B, 84(24):245450, December 2011.

[15] A. A. Joshi and A. Majumdar. Transient ballistic and diffusive phonon heat transport in thin films. Journal of Applied Physics, 74(1):31, 1993.

[16] S. V. J. Narumanchi, J. Y. Murthy, and C. H. Amon. Submicron heat transport model in silicon accounting for phonon dispersion and polarization. Journal of Heat Transfer, 126(6):946, 2004.

[17] J. Loy, D. Singh, and J. Murthy. Non-Gray phonon transport using a hybrid BTE-Fourier solver. 2009 ASME International Summer Heat Transfer Conference, (Md):1-10, 2009.

[18] D. Singh, J. Y. Murthy, and T. S. Fisher. Frequency resolved phonon transport in Si/Ge nanocomposites. ASME 2011 Pacific Rim Technical Conference, 2013.

[19] T. O. Woodruff and H. Ehrenreich. Absorption of sound in insulators. Physical Review, 123(5), 1961.

[20] T. Soma, H. Matsuo, and Y. Saitoh. Volume-dependence of phonon frequencies and mode grneisen parameters for si and ge. Solid State Communications, 39(11):1193 - 1197, 1981.

[21] D. Broido, A. Ward, and N. Mingo. Lattice thermal conductivity of silicon from empirical interatomic potentials. Physical Review B, 72(1):014308, July 2005.

[22] G.-M. Rignanese, J.-P. Michenaud, and X. Gonze. Ab initio study of the volume dependence of dynamical and thermodynamical properties of silicon. Physical Review B, 53(8):4488-4497, February 1996. 
[23] A. A. Kiselev and G. J. Iafrate. Phonon dynamics and phonon assisted losses in Euler-Bernoulli nanobeams. Physical Review B, 77(20):205436, May 2008.

[24] J. Tersoff. Empirical interatomic potential for silicon with improved elastic properties. Physical Review B, 38(14):9902-9905, November 1988.

[25] D. Brenner. Empirical potential for hydrocarbons for use in simulating the chemical vapor deposition of diamond films. Physical Review B, 42(15):9458-9471, November 1990.

[26] F. H. Stillinger and T. A. Weber. Computer simulation of local order in condensed phases of silicon. Physical Review B, 31(8):5262-5271, April 1985.

[27] H. Zhao, Z. Tang, G Li, and N. R. Aluru. Quasiharmonic models for the calculation of thermodynamic properties of crystalline silicon under strain. Journal of Applied Physics, 99(6):1-12, 2006.

[28] Z. Tang, H. Zhao, G. Li, and N. R. Aluru. Finite-temperature quasicontinuum method for multiscale analysis of silicon nanostructures. Physical Review B, 74(6):064110, August 2006.

[29] P. E. Hopkins. Dispersion considerations affecting phonon-mass impurity scattering rates. AIP Advances, 1(4):041705, December 2011.

[30] M. Asheghi, K. Kurabayashi, R. Kasnavi, and K. E. Goodson. Thermal conduction in doped singlecrystal silicon films. Journal of Applied Physics, 91(8):5079, 2002.

[31] J. M. Ziman. The effect of free electrons on lattice conduction. Philosophical Magazine, 1(14):191, 1955.

[32] M. G. Holland. Analysis of lattice thermal conductivity. Physical Review, 132(6):2461-2471, 1963.

[33] J. Callaway. Model for lattice thermal conductivity at low temperatures. Physical Review, 113(4):1046, 1959.

[34] Y.-J. Han and P. G. Klemens. Anharmonic thermal resistivity of dielectric crystals at low temperatures. Physical Review B, 48(9):6033-6042, September 1993.

[35] P. G. Klemens. Anharmonic decay of optical phonons. Physical Review, 148(2):845-848, August 1966.

[36] G Chen. Size and interface effects on thermal conductivity of superlattices and periodic thin-film structures. Journal of Heat Transfer, 119(2):220-229, 1997. 
[37] S. Li, H. Chu, and W. Yan. Numerical study of phonon radiative transfer in porous nanostructures. International Journal of Heat and Mass Transfer, 51(15-16):3924-3931, July 2008.

[38] H. Li, Y. Yu, and G. Li. Computational modeling and analysis of thermoelectric properties of nanoporous silicon. Journal of Applied Physics, 115(12):124316, March 2014.

[39] J. H. Prevost. Finite element analysis of dynamic coupled thermoelasticity problems with relaxation times. Journal of Applied Mechanics, 50(December):817-822, 1983.

[40] A. Duwel, R. N. Candler, T. W. Kenny, and M. Varghese. Engineering MEMS resonators with low thermoelastic damping. Journal of Microelectromechanical Systems, 15(6):1437-1445, December 2006.

[41] Y. Yi and M. Matin. Eigenvalue solution of thermoelastic damping in beam resonators using a finite element analysis. Journal of Vibration and Acoustics, 129(4):478-483, 2007.

[42] E. Serra and M. Bonaldi. A finite element formulation for thermoelastic damping analysis. International Journal for Numerical Methods in Engineering, 78(6):671-691, 2009.

[43] Y. Xu and G. Li. Strain effect analysis on phonon thermal conductivity of two-dimensional nanocomposites. Journal of Applied Physics, 106(11):114302, 2009.

[44] Z. Zhu, D. A. Romero, D. P. Sellan, A. Nabovati, and C. H. Amon. Assessment of the Holland model for silicon phonon-phonon relaxation times using lattice dynamics calculations. Journal of Applied Physics, 113(17):173511, May 2013.

[45] S. Wei, C. Li, and M. Chou. Ab initio calculation of thermodynamic properties of silicon. Physical Review B, 50(19):14587-14590, November 1994.

[46] B. A. Weinstein and G. J. Piermarini. Raman scattering and phonon dispersion in Si and GaP at very high pressure. Physical Review B, 12(4):1172-1186, August 1975.

[47] Z. Tang, Y. Xu, G. Li, and N. R. Aluru. Physical Models for Coupled Electromechanical Analysis of Silicon Nanoelectromechanical Systems. Journal of Applied Physics, 97(11):114304, 2005.

[48] Subhadeep De, K. Kunal, and N. R. Aluru. Mixed role of surface on intrinsic losses in silicon nanostructures. Journal of Applied Physics, 119(11):114304, 2016. 
[49] K. Kunal and N. R. Aluru. Intrinsic dissipation in a nano-mechanical resonator. Journal of Applied Physics, 116(9):094304, September 2014.

[50] A. Balandin and K. L. Wang. Effect of phonon confinement on the thermoelectric figure of merit of quantum wells. Journal of Applied Physics, 84(11):6149, 1998. 